Check for updates

Cite this: J. Mater. Chem. A, 2021, 9 , 23419

Received 21st June 2021

Accepted 11th October 2021

DOI: $10.1039 / \mathrm{d} 1 \mathrm{ta0} 2 \mathrm{242j}$

rsc.li/materials-a

\section{The impact of ligands on the synthesis and application of metal halide perovskite nanocrystals}

\begin{abstract}
Fatima Haydous, (D) James M. Gardner (D)* and Ute B. Cappel (D)*
Metal halide perovskites have emerged as attractive materials for use in solar cells, light emitting diodes and other optoelectronic devices, mainly due to their impressive charge transport properties, strong light absorption, long carrier diffusion lengths and long excited state lifetime. The extensive research on these materials has paved the way for a new class of materials: metal halide perovskite nanocrystals (NCs). Due to their high photoluminescence quantum yield and narrow emission that can be tuned by size and compositional variations, perovskite NCs are considered to be ideal candidates compared to traditional quantum dots. With the growing interest in these materials and the current challenges in their commercialization, this review aims mainly to provide the necessary understanding of the influence of capping ligands on the synthesis and application of perovskite NCs. The different synthetic approaches and the role of ligands in determining the morphological and optical properties of the resulting NCs will be discussed. Thereafter, we review the advances in understanding the surface chemistry and ligation in the metal halide perovskite NCs. Lastly, we review the ligand exchange and management processes that are shown to be beneficial in improving the performance and stability of perovskite nanocrystal films for optoelectronic applications.
\end{abstract}

\section{Introduction}

During the last decades, nanoscale materials have become essential in our daily lives, as they are embraced in most of our daily practices ${ }^{\mathbf{1 - 3}}$ including cosmetics, ${ }^{\mathbf{4 , 5}}$ biological applications
Division of Applied Physical Chemistry, Department of Chemistry, KTH - Royal Institute of Technology, SE-100 44 Stockholm, Sweden. E-mail: jgardner@kth.se; cappel@kth.se

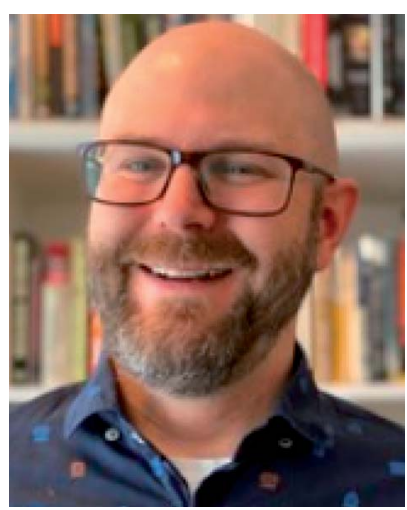

Dr James $M$ Gardner received his PhD in Physical, Inorganic, and Materials Chemistry from The Johns Hopkins University in 2009. He joined the faculty of the Department of Chemistry at KTH Royal Institute of Technology in 2011 as an Assistant Professor. Since 2016, he has been the Associate Professor of Photoelectrochemistry in the Division of Applied Physical Chemistry at KTH. That same year, James became Deputy Head of the Center for Molecular Devices (CMD). The CMD is a collaborative research environment between KTH and Uppsala University that focuses on all aspects of emerging solar cell technologies. His current research interests focus on photochemistry and photophysics of interfaces. Of particular interest is understanding the energetics, kinetics, and conditions that promote interfacial charge transfer chemistry as this relates to dye-sensitized solar cells and perovskite solar cells. 
(e.g. biosensors) ${ }^{6-8}$ electronic and optoelectronic devices (like smart phones and displays). ${ }^{9-11}$ The reason behind the interest in these materials and their great potential in all of these applications are their outstanding properties due to their large surface-to-volume ratio in comparison to their corresponding bulk materials. Semiconductor nanocrystals (NCs) can exhibit unique properties such as high extinction coefficients, rapid charge separation, good chemical/thermal/environmental stability, and ease of synthesis. Typically, the solution-based synthesis of NCs requires surface passivating molecules known as capping ligands, which bind to the surface of the NCs to control crystal size and isolate them from their environment. The produced NCs have versatile surface chemistries that allow for dispersion in different solvents and which enable their integration into various matrices and devices. Another key advantage is the quantum confinement effect, in which the electron and hole are confined in the NC. This is primarily observed in quantum dots (QDs), which are semiconductor NCs with a size usually in the range of $2-10 \mathrm{~nm}$ that confines electron-hole pairs in all three dimensions. Due to this effect, these NCs exhibit discrete energy levels similar to those of atoms or molecules and the light absorption and energy levels can be tuned through the size of the crystals. ${ }^{\mathbf{1 2}}$

In parallel, the continued research on new materials has led to novel solutions for overcoming the increasing global energy demands. Even though metal halide perovskites (MHPs) were discovered more than 40 years ago, the interest in these materials was stimulated by the 2012 reports in which MHPs were successfully used as light absorbers in solar cells. ${ }^{13-15}$ The attractive properties of MHPs including the long carrier diffusion lengths, strong light absorption, impressive charge transport and tuneable bandgap through compositional variations promoted a vast amount of research in this field..$^{15,16}$ Due to the fast developments in the application of MHPs in solar cells, a certified record efficiency more than $25 \%$ was achieved within only one decade of development compared to several decades for other solar cell technologies. ${ }^{17}$ MHPs are appealing

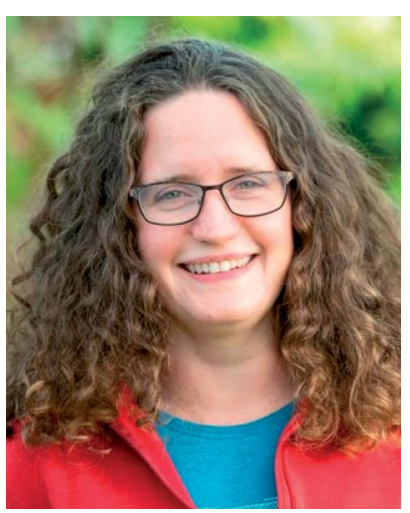

Dr Ute Cappel received her PhD in Chemistry at Uppsala University in 2011. Following a MarieCurie fellowship at Imperial College London, she was a researcher at the Department of Physics and Astronomy at Uppsala University. In 2017, she became an Assistant Professor at the Department of Chemistry at KTH Royal Institute of Technology and was promoted to Associate Professor in 2020. Her research focuses on the characterization of materials for and processes in hybrid solar cells, in particular lead halide perovskites and quantum dots. She uses synchrotron-based photoelectron spectroscopy to study the chemical and electronic structure of surfaces and interfaces. materials not only in photovoltaics but also in LEDs, photodetectors, lasers and other optoelectronic applications, where a significant progress had been also reported. ${ }^{\mathbf{1 8 - 2 4}}$

Researchers have shown that a synergetic effect can be realized by combining the advantages of these two classes of materials: perovskites and nanocrystals. ${ }^{25}$ Extensive research has therefore concentrated on the synthesis, characterization and application of MHP NCs and particularly lead halide perovskite NCs, which may substitute traditional metal chalcogenide NCs in some applications. ${ }^{26}$ Perovskite NCs exhibit intriguing properties ranging from low fabrication cost, simple and facile synthesis, outstanding photoluminescence quantum yields (PLQY) and narrow emission. Their bandgap can be tuned to match the whole visible light region via composition modification and crystal size control. ${ }^{26,27}$ Moreover, compared to bulk MHPs, NCs are considered beneficial in several aspects. Firstly, surface effects can stabilize the cubic perovskite crystal structure, which would be unstable as the bulk material. ${ }^{\mathbf{1 3}}$ Secondly, NCs show enhanced PLQY compared to their bulk counterparts with PLQY limited by mobile ionic defects and small exciton binding energy. ${ }^{\mathbf{1 4}}$ These properties make them interesting materials for next generation photovoltaic and light emitting devices.

With the rapid advances in this field, there have been several reviews during the previous years revealing the potential of MHPs in the form of NCs. ${ }^{9,13,14,16,28}$ The main focus of these reviews was the synthesis of the perovskite NCs and their applications. Recently, more specific reviews have been published encountering precise issues related to metal halide perovskite nanocrystals, such as doping with lanthanide, ${ }^{29}$ using mesoporous matrices as hosts for the nanocrystals ${ }^{30}$ and lead-free halide nanocrystals. ${ }^{31}$ Even though ligands have a profound effect on the growth and morphological, structural and optoelectronic properties perovskite NCs, the type of interaction between the perovskite nanocrystals and capping ligands has been significantly less discussed. Thus, this review will shed light on the surface chemistry of the perovskite nanocrystals and the role of ligand modification in determining the growth, optical and morphological properties of the nanocrystals and therefore their application.

Based on that, the review is divided into five main parts. The first part addresses ligation and the different types of ligands used in the synthesis of MHP NCs. The second part gives a brief overview of the structural and optoelectronic properties of MHP NCs and their stability. In the third part, a summary of the different synthesis approaches of MHP NCs will be given with the focus on the influence of ligands in controlling the morphology and composition of the NCs. The fourth part will describe the surface chemistry of MHP NCs and the NC-ligand interaction and its influence on the purification procedure of the NCs. The last section will detail the ligand management procedures that have been applied to improve the performance and stability of optoelectronic devices based on MHP NC films. Finally, we will conclude the review by discussing the remaining open issues and providing an outlook on the future directions in this field. 


\section{Ligands}

Ligands are molecules or ions that bind to a metal atom or ion forming a coordination complex. These molecules or ions are usually used in the synthesis of NCs, where they bind to the NCs surface to stabilize them and compensate for their high surface to-volume ratio. In this section we will introduce the most common ligands for MHP NCs and their binding types.

The bonding between ligands and metals varies from covalent to ionic bonding. In this regard, the different types of bonding between the ligands and surface states of the NCs can be described using the Covalent Bond Classification (CBC) that was proposed initially by Green $^{32}$ to describe metal-organic complexes. Based on this model, ligands can be divided in three categories: a Z-type ligand with an empty orbital that acts as a Lewis acid accepting an electron pair from an anion site at the NC surface, an L-type ligand which is considered as a Lewis base with a filled orbital that donates two electrons to an unoccupied surface metal orbital and an X-type ligand which forms a covalent bond with a singly occupied orbital on the surface. ${ }^{33,34}$

Fig. 1 shows an overview of different types of ligands that have been used for stabilizing MHP NCs and that will be considered in this review.

In general, two types of ligands are used in the synthesis of MHP NCs: carboxylic acids which prevent coagulation of the NCs through steric repulsion and amines that are integral in controlling the crystallization of the NCs. ${ }^{35}$ Such monodentate ligands consist of a long alkyl tail or a phenyl group and a polar head which binds to an atom or ion at the surface of the NC.
NCs can be also capped with bidentate and multidentate ligands which can bind to two or more atoms or ions at the NC surface. These ligands have a stronger binding to the NC surface compared to the monodentate ones, which leads to less structural distortions at the NC surface and as a consequence stabilizing the NCs more effectively. ${ }^{36,37}$

Besides the aforementioned ligands, branched ligands have been investigated as they enable a better control of the NC size and uniformity compared to ligands with straight chains. Another type of ligand is the zwitterionic ligand, which has several functional groups at the same end of the molecule that can anchor simultaneously to the NC surface. As a result, these ligands have a stronger interaction with the NC surface in comparison to ligands with one coordinating group. Moreover, ligands can be anions (such as halides, $\mathrm{S}^{2-}, \mathrm{SCN}^{-}$and $\mathrm{NH}_{2}{ }^{-}$) or cations (such as $\mathrm{NO}^{+}$and $\mathrm{N}_{2} \mathrm{H}_{5}{ }^{+}$). ${ }^{38-44}$ Indeed, as will be discussed later, the carboxylic acid and amine ligands used in the synthesis of MHP NCs bind to the NCs surface as carboxylate anions and ammonium cations, respectively. These ionic ligands influence the nature of trapping sites at the surface of the NCs and passivate them to improve the photoluminescence and stability of NCs.

\section{Perovskite nanocrystals}

In this section, we discuss the structural and optoelectronic properties and the stability of perovskite NCs. These properties will be addressed throughout the review while discussing the influence of ligands on the synthesis and application of MHP NCs.

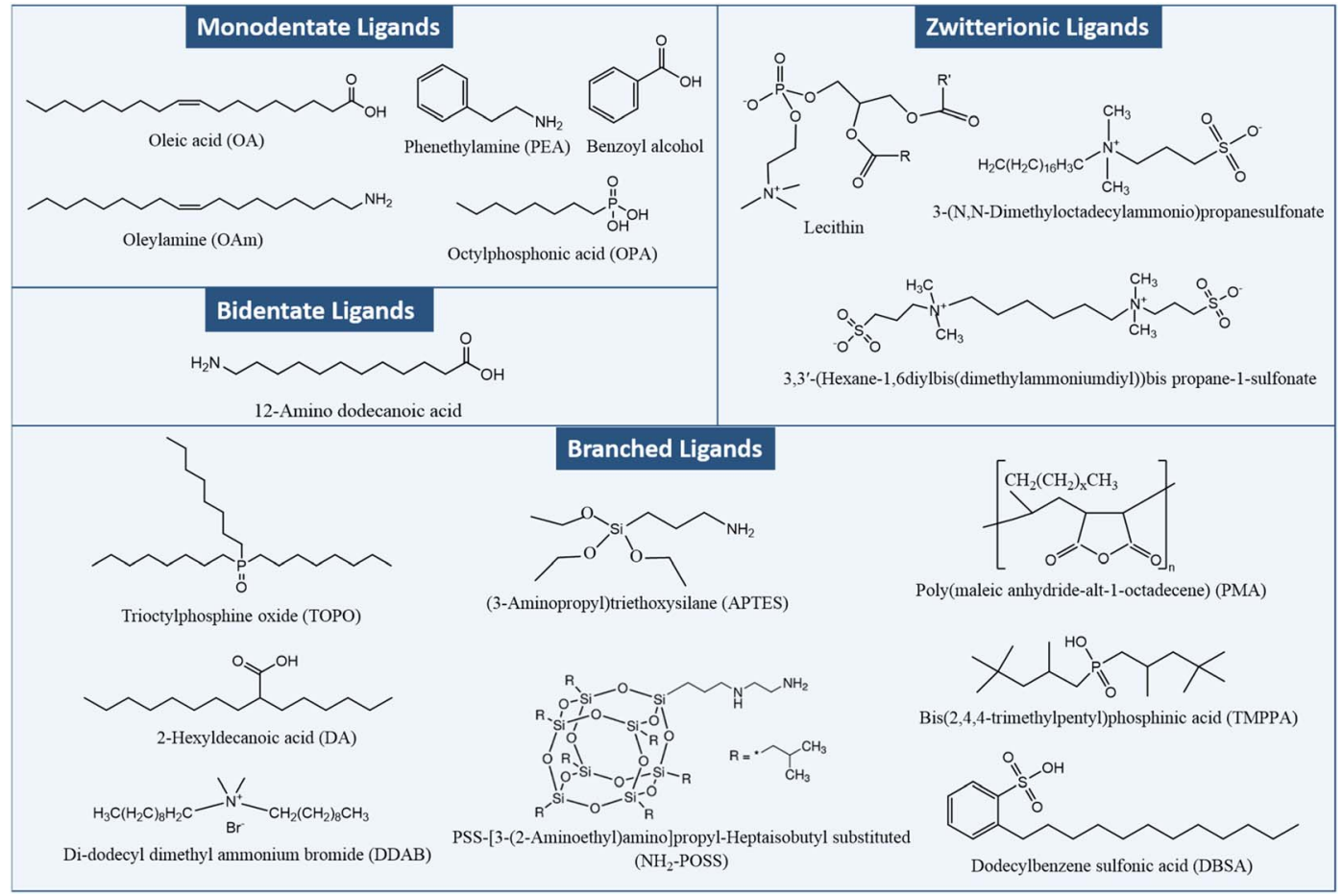

Fig. 1 Chemical structures of different types of capping ligands for MHP NCs. 


\subsection{Crystal structure}

Analogous to oxide perovskites, MHPs of the overall $\mathrm{ABX}_{3}$ formula (with A being a large monovalent cation, $\mathrm{B}$ an inorganic-metal cation and $\mathrm{X}$ a halide anion such as $\mathrm{Cl}^{-}, \mathrm{Br}^{-}$, $\mathrm{I}^{-}$) consist of a three-dimensional (3D) network of corner sharing $\mathrm{BX}_{6}$ octahedra (Fig. 2a). In the cubic void between the corner sharing octahedra one or more A cation forms ionic bonds to the anionic $\mathrm{BX}_{6}{ }^{4-}$ network. Most of the studied MHP $\mathrm{NCs}$ are based on $\mathrm{Pb}^{2+}$ at the B-site. These lead halide perovskite (LHP) NCs can be classified depending on the type of the A cation into three groups: hybrid organic-inorganic, allinorganic and mixed perovskites. Hybrid organic-inorganic LHP NCs contain an organic ammonium cation at the A-site of the perovskite such as: methylammonium $\left(\mathrm{MA}^{+}\right)$and formamidinium $\left(\mathrm{FA}^{+}\right)$. All-inorganic lead halide perovskites are isostructural to the hybrid perovskite with A being an inorganic cation such as $\mathrm{Cs}^{+}$or $\mathrm{Rb}^{+}$. In mixed LHP NCs, the composition of the A cation can be adjusted by using different ammonium cations $^{45,46}$ or by incorporating both inorganic and organic cations. ${ }^{47}$

Alternative to LHP NCs, MHP NCs based on more environmentally friendly metal cations at the B-site $\left(\mathrm{Sn}^{2+}, \mathrm{Ge}^{2+}, \mathrm{Sb}^{3+}\right.$, $\mathrm{Bi}^{3+}, \mathrm{In}^{3+}, \mathrm{Cu}^{+}$or $\mathrm{Ag}^{+}$) replacing $\mathrm{Pb}^{2+}$ became highly desirable. However, these lead-free perovskite NCs suffer from oxidative instability, indirect or large band gaps, and synthesis

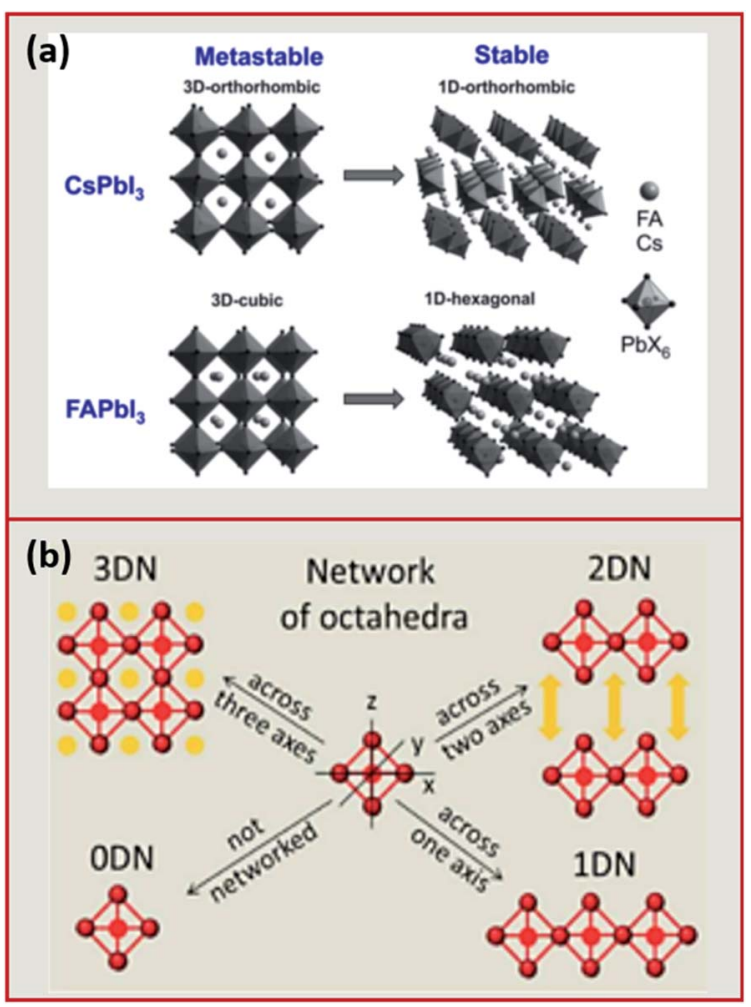

Fig. 2 (a) Illustration of the 3D to 1D phase transition in $\mathrm{CsPb}_{3}$ and $\mathrm{FAPbl}_{3}$. Reproduced with permission from ref. 27. (b) Schematic presenting the differences between the different dimensionalities (ODN, $1 D N, 2 D N$ and 3DN) depending on the connectivity of the octahedra. Reproduced with permission from ref. 56. difficulties since they compete with more thermodynamically favourable phases. ${ }^{13}$ In addition, they still show inferior properties compared to Pb-based perovskite NCs (i.e. less defect tolerance and hence lower PLQY). ${ }^{31}$

MHPs are typically observed in three 3D polymorphs: cubic, tetragonal and orthorhombic with the cubic phase existing at the highest temperature. ${ }^{13}$ The stability of the perovskite phases can be predicted using the Goldschmidt tolerance $(t)$ and the octahedral factor $(\mu)$ factors. ${ }^{48-50}$ The latter is determined from the ratio of the ionic radii of the $\mathrm{B}$ cation $\left(r_{\mathrm{B}}\right)$ over the halide $\left(r_{\mathrm{X}}\right)$ such that: $\mu=r_{\mathrm{B}} / r_{\mathrm{X}}$. When this factor ranges between 0.41 and 0.89 , the $\mathrm{BX}_{6}{ }^{4-}$ octahedra, and thereby the perovskite structure, are considered stable..$^{51}$ On the other hand, the tolerance factor depends additionally on the ionic radii of the A-site cation $\left(r_{\mathrm{A}}\right)$ according to the following equation:

$$
t=\frac{\left(r_{\mathrm{A}}+r_{\mathrm{X}}\right)}{\sqrt{2}\left(r_{\mathrm{B}}+r_{\mathrm{X}}\right)}
$$

This factor has been extensively used to predict the stability of perovskite structures through assessing if the A-site cation fits in the voids of the framework. ${ }^{48,49}$ For $t$ values between 0.8 and 1.0, the octahedra are stable resulting in a stable perovskite structure. The perovskite is cubic when $t$ is close to one, otherwise, the octahedra will partially tilt resulting in orthorhombic or tetragonal structures. Only the cubic and tetragonal 3D polymorphs are interesting for optoelectronic applications as they are photoactive exhibiting the desired semiconducting properties. ${ }^{49,50}$ As an example, the $3 \mathrm{D}$ polymorphs, of $\mathrm{CsPbI}_{3}$ and $\mathrm{FAPbI}_{3}$ are metastable at room temperature (RT). This is because the size of A cations for these two near-IR emitting perovskites are at the borderline for optimal 3D perovskite structure; with $\mathrm{Cs}^{+}$ion being too small $\left(t \sim 0.89\right.$ for $\left.\mathrm{CsPbI}_{3}\right)$ and $\mathrm{FA}^{+}$being too large $\left(t \sim 1\right.$ for $\left.\mathrm{FAPbI}_{3}\right){ }^{27,50,52}$ Therefore, these two materials easily undergo a phase transition at RT to their more stable phases; $\mathrm{FAPbI}_{3}$ to a one-dimensional (1D) hexagonal phase and $\mathrm{CsPbI}_{3}$ to a $1 \mathrm{D}$ orthorhombic phase (illustrated in Fig. $2 \mathrm{a}^{27,48}$ ). These two phases, known as "yellow phases" have wider band gaps than the $3 \mathrm{D}$ motifs and show poor electron transport which limits the full utilization of $\mathrm{CsPbI}_{3}$ and $\mathrm{FAPbI}_{3}$ in devices. ${ }^{\mathbf{1 3 , 5 0}}$ Nonetheless, it was shown that the polymorph stability of MHPs can be adjusted in NCs because of the governing surface effects. The cubic phase of $\mathrm{CsPbI}_{3}$ was shown to be stabilized at RT in ambient air for several months in $\mathrm{CsPbI}_{3}$ QDs and QD films due to the contribution of surface energy. ${ }^{50,53}$ In this way, the under-coordinated surface sites or bound ligands can affect bonding geometries for the entire crystal.

In addition to the 3D MHP polymorphs, low dimensional NCs have been synthesized by tailoring the composition of the MHPs. ${ }^{31,54}$ These NCs exhibit a variety of shapes such as: zerodimensional (0D) QDs, one-dimensional (1D) nanowires (NWs) or nanorods (NRs) and two-dimensional (2D) nanosheets (NSs) or nanoplatelets (NPLs). ${ }^{50,55}$ Compared to the corner sharing octahedra in 3D perovskites, the connectivity of the octahedra in the lower dimensions is presented in Fig. 2b. The low dimensional NCs are usually more stable than 3D NCs 
against environmental factors such as moisture. ${ }^{56}$ However, the 3D polymorphs of MHPs are more interesting than the $2 \mathrm{D}$ and 1D motifs because they exhibit more appealing semiconductor properties (e.g. suitable bandgap and good electron transport) that are desirable for optoelectronic applications. ${ }^{\mathbf{5 0}}$

\subsection{Optoelectronic properties}

In this part, we discuss the different optoelectronic properties of MHP NCs that are of interest for their application in devices. These properties, as will be shown in Sections 5 and 6, are affected by the ligands binding to the NCs and therefore can be tailored depending on the application of the NCs via ligand control.

3.2.1 Absorption and emission. Following the pioneering study by Protesescu et al. ${ }^{57}$ on the synthesis of $\mathrm{CsPbX}_{3}$ NCs with variable halide content, the outstanding optical properties of LHP NCs, and MHP NCs in general, have been demonstrated. The absorption edge and photoluminescence (PL) peak shifted to the red when increasing the size of the halide anion, such that $\mathrm{CsPbCl}_{3}$ emitted at the blue edge of the visible spectrum, $\mathrm{CsPbBr}_{3}$ in the green and $\mathrm{CsPbI}_{3}$ at the red edge. As shown in Fig. 3a, the absorption and emission of the NCs can be further tuned over the entire visible range by using halide mixtures and changing their ratios in $\mathrm{CsPbX}_{3} \mathrm{NCs}^{57}$ Similarly, the variation of the halide content from $\mathrm{Cl}$ to $\mathrm{Br}$ to I red shifted the emission in MA perovskite $\mathrm{NCs}^{58}$ and FA-based perovskite NCs. ${ }^{59-61}$ This tunability is due to the electronic structure of the lead halide perovskites that is presented in Fig. $3 \mathrm{~b}$. The conduction band (CB) is formed from the antibonding mixing between $\mathrm{Pb} 6 \mathrm{p}$ orbitals and halide outer $\mathrm{p}$ orbitals with the major contribution from $\mathrm{Pb} 6 \mathrm{p}$. The valence band (VB) is composed of the antibonding orbitals from the hybridization of the halide valence $\mathrm{p}$ orbitals with $\mathrm{Pb} 6 \mathrm{~s}$ orbitals. ${ }^{62,63}$ Thus, the halide composition determines the energy of the halide antibonding orbital resulting in a shift in the VB edge and a slight modification of the CB edge (Fig. 3c). ${ }^{63}$

The optical properties of the MHP NCs are also influenced by the cation at the B-site of the perovskite. For instance, the band gap was shown to be red shifted when replacing $\mathrm{Pb}^{2+}$ with $\mathrm{Sn}^{2+}$ most probably because of the higher electronegativity of the latter cation. ${ }^{64}$ Other examples include partial substitution of $\mathrm{Pb}^{2+}$ or complete substitution forming lead-free MHP NCs that modify the optical properties of their LHP alternates. ${ }^{31,65}$

Even though the A cation of the perovskite does not contribute to the density of states (DOS) of the CB and VB, it can influence the band gap. ${ }^{66}$ The decrease of the size of the A cation (for example from $\mathrm{FA}^{+}$to $\mathrm{MA}^{+}$to $\mathrm{Cs}^{+}$) was shown to increase the band gap of LHP. ${ }^{66}$ This is ascribed to the increase in the tilting angle of the $\mathrm{Pb}-\mathrm{X}-\mathrm{Pb}$ bonding that is associated to the distortion of the cubic crystal structure. ${ }^{67}$

Perovskite NCs have narrow emissions with line widths that are less than $100 \mathrm{meV}$ which is essential for lighting applications. ${ }^{57}$ Also, compared to their bulk counterparts, MHP NCs exhibit extremely high PLQY values. ${ }^{60}$ PLQY of nearly $100 \%$ have been observed for caesium and methylammonium lead bromide or iodide NCs and values of 70-90\% have been achieved for $\mathrm{FA}^{+}$-based NCs. ${ }^{6-73}$ In comparison, chloride-based perovskite NCs exhibit lower PLQY (below 20\%) because of the changes in the crystal structure associated with the smaller size of $\mathrm{Cl}^{-}$, and the non-radiative trapping sites due to defects that are less shallow compared to other halide perovskites. ${ }^{\mathbf{1 4}}$

3.2.2 Quantum confinement. Besides compositional modifications, quantum-size effects have a major role in tuning the band gap of MHP NCs. ${ }^{30}$ To achieve quantum confinement, the size of the NC should be similar to or smaller than the Bohr exciton radius, which is the most probable delocalization distance of an exciton in the bulk semiconductor. This confinement results in a blue shift of the absorption and emission of the NC with respect to its bulk form. The resulting confinement energy that depends on the size of the NC determines the extent of the blue shift. ${ }^{57}$ This is shown in Fig. 4a where the $\mathrm{PL}$ emission of $\mathrm{CsPbBr}_{3}$ nanocubes blue shifts with decreasing the size of the NCs.

The Bohr diameter of Wannier-Mott type excitons, estimated according to the effective mass approximation, was found to be 5,7 and $12 \mathrm{~nm}$ for $\mathrm{CsPbCl}_{3}, \mathrm{CsPbr}_{3}$ and $\mathrm{CsPbI}_{3}$

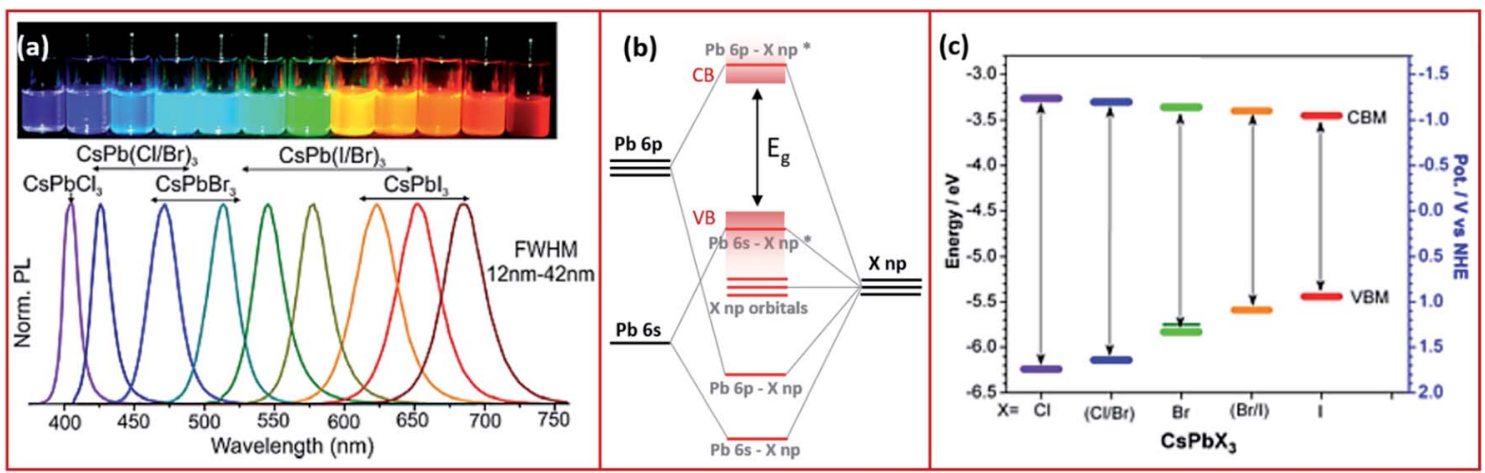

Fig. 3 (a) The PL spectra of $\mathrm{CsPbX}_{3}$ NCs with variable halide content with the corresponding image of the different NC solutions in toluene under UV illumination. Reproduced with permission from ref. 57. (b) A schematic representing the electronic structure of lead halide perovskites. (c) Band edge energies (conduction band CBM and valence band VBM) of $\mathrm{CsPbX}_{3} \mathrm{NCs}$ extracted from cyclic voltammetry measurements with a scan rate of $50 \mathrm{mV} \mathrm{s}^{-1}$ of the NCs dispersed in tetra-butyl ammonium perchlorate electrolyte in a mixture of acetonitrile and toluene. Modified with permission from ref. 63 . 


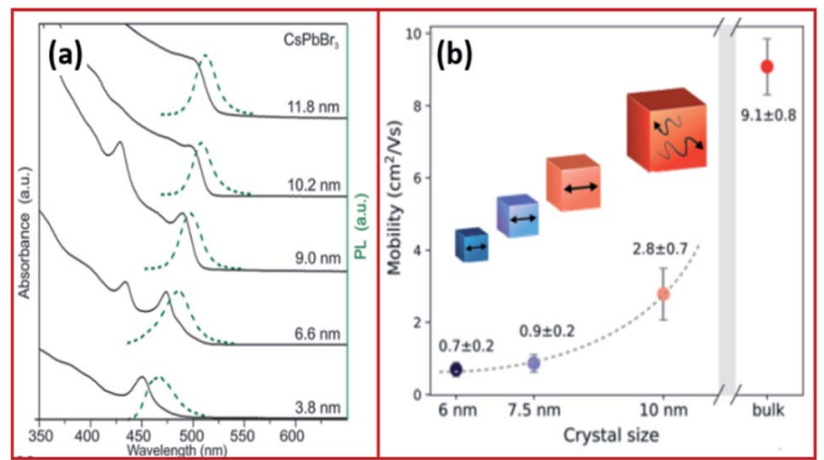

Fig. 4 (a) Absorption and emission of $\mathrm{CsPbBr}_{3} \mathrm{NCs}$ with variable size demonstrating quantum-size effects. (b) Size dependence of effective charge-carrier mobility of $\mathrm{CsPbBr}_{3}$. Panels (a) and (b) are reproduced with permission from ref. 57 and 81 , respectively.

NCs and 1.5-3 nm for $\mathrm{MAPbBr}_{3}$ and $\mathrm{MAPbI}_{3} \mathrm{NCs}^{57,74,75}$ As a result, the confinement in LHP NCs which are typically in the form of 4-15 nm nanocubes is usually negligible or very low. For instance, the exciton binding energies of $\mathrm{CsPbCl}_{3}, \mathrm{CsPbBr}_{3}$, $\mathrm{CsPbI}_{3}$ nanocubes were determined to be 75,40 and $20 \mathrm{meV}$, respectively, which are comparable to those of their bulk counterparts and to $\mathrm{MAPbI}_{3}$ with an exciton binding energy of $\leq 25 \mathrm{meV} \cdot{ }^{57,76-79}$ However, in the case of nanosheets and nanowires, the confinement becomes more significant due to the use of long chain ligands, which enable the synthesis of very thin nanosheets (few monolayers) and nanowires of small diameters (less than $10 \mathrm{~nm}$ ). A high exciton binding energy of $120 \mathrm{meV}$ was measured for $3.4 \mathrm{~nm}$ thick $\mathrm{CsPbBr}_{3}$ nanoplatelets demonstrating a strong confinement in one dimension. ${ }^{76,80}$

3.2.3 Charge carrier dynamics. The charge transport mechanism in MHP NCs is expected to be different from the bulk. Indeed, it was found that charge carriers in films based on $\mathrm{CsPbBr}_{3}$ NCs have a more localized nature compared to polycrystalline films. As seen in Fig. 4b, the effective charge-carrier mobility decreased with decreasing size of the crystal confirming the localization of charge carriers within the NCs because of increased excitonic effects and increased proximity of the barriers between the NCs. ${ }^{81}$

Studying the PLQY values of perovskite NCs along with their PL decay dynamics gives insights regarding the radiative and non-radiative recombination processes and therefore understanding the defects and trap states in these NCs. ${ }^{30}$ As in any semiconductor material, the lifetime of excited states in MHP NCs is usually shorter than that of its bulk counterpart. In the case of inorganic $\mathrm{CsPbX}_{3} \mathrm{NCs}$, time-resolved PL measurements show a bi-exponential decay with a radiative lifetime in the nanosecond range between 1 and $29 \mathrm{~ns}$. The PL lifetime of these NCs increases with the decrease of the band gap. ${ }^{13,57,82}$ In comparison, organic LHP NCs exhibit longer lifetimes with $\mathrm{FAPbI}_{3}, \mathrm{MAPbI}_{3}$ and $\mathrm{MAPbBr}_{3-x} \mathrm{Cl}_{x}(x=0.6-2) \mathrm{NCs}$, for instance, showing decay lifetimes of 70, 100 and 40-400 ns, respectively. ${ }^{48,57}$ Mixed organic-inorganic LHP NCs such as $\mathrm{FA}_{0.1} \mathrm{Cs}_{0.9} \mathrm{PbI}_{3}$ exhibit radiative lifetimes of $51 \mathrm{~ns} .^{48}$ Lead-free MHP NCs show comparable PL decay lifetimes to LHP NCs. ${ }^{31}$
3.2.4 Defect tolerance and surface passivation. Among the most attractive features of MHP which contribute to their high performance in photovoltaics and their bright emission is their defect tolerance. ${ }^{83}$ The so-called defect tolerance is the ability of retaining the electronic structure regardless of a high concentration of defects in the material. ${ }^{\mathbf{1 4}}$ It has been shown that in halide perovskites under stoichiometric conditions, Schottkytype point defects that are intrinsic cation and anion vacancies are the main defects due to their low formation energy. ${ }^{84}$ As shown in Fig. 5a, interstitial and anti-site point defects, which tend to form deep trap states have higher formation energies and are therefore almost absent in perovskites. ${ }^{28}$ Besides, the low lattice energy in these materials promotes other types of defects such as dislocations, grain boundaries and twin planes. ${ }^{13}$ Studies have shown that despite these defects in LHPs along with the surface defects in the high surface area NCs, the optoelectronic properties of the LHP NCs are not altered since they do not form mid-gap trap states and induce rather shallow states near the energy bands. ${ }^{85-87}$ Shallow defects do not affect the charge carrier transport and the radiative recombination in these NCs. ${ }^{30}$ This defect tolerance in MHP NCs is the reason of their very bright photoluminescence and their efficient utilization in solar cells and LED devices without the need of electronic surface passivation as in the case of metal chalcogenide QDs. ${ }^{13,57}$ The differences between MHPs and the conventional semiconductors in terms of the effect of defects on their electronic properties is illustrated in Fig. 5 b..$^{13}$

\subsection{Stability}

With all the promising optoelectronic properties and the achieved progress in the synthesis of MHP NCs, the full implementation of their potential remains restricted by their limited stability. MHP NCs are highly vulnerable to polar solvents, which threaten their optical properties and long-term structural integrity. ${ }^{\mathbf{1 3 , 8 8}}$ Yuan et al., ${ }^{89}$ for instance, showed that the use of certain antisolvents in the postsynthetic cleaning steps of $\operatorname{CsPbBr}_{1-x} \mathrm{I}_{x}$ perovskite NCs can modify their halide content and thereby their overall optical properties.

In addition, MHP materials exhibit poor chemical stability and are highly sensitive to moisture, light, and elevated temperatures. This was shown to limit the practical application

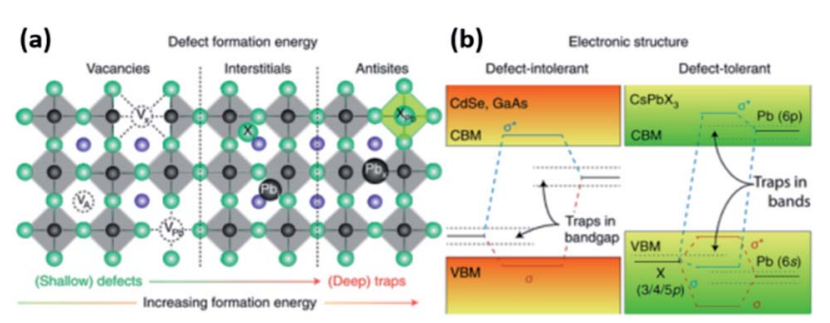

Fig. 5 (a) The typical point defects present in LHPs ordered with respect to their formation energies and positions in the band gap. (b) Schematic illustration showing the differences in the electronic structures of defect intolerant semiconductors (such as metal chalcogenides) and defect tolerant MHPs. Reproduced with permission from ref. 28 
of bulk MHPs in highly efficient solar cells. ${ }^{\mathbf{9 0 9 1}}$ The stability of the hybrid $\mathrm{MAPbI}_{3}$, for example, is influenced by its decomposition into volatile $\mathrm{HI}$ and $\mathrm{CH}_{3} \mathrm{NH}_{2} \cdot{ }^{13}$ In addition, MHP NCs can degrade in the presence of a combination of light, and oxygen because of the diffusion of photogenerated $\mathrm{O}_{2}{ }^{-}$anion into the halide vacancy sites promoting the oxidation of the perovskite. ${ }^{92}$ Moreover, of certain concern is the thermal instability of LHP NCs because of their low melting point where sintering of films based on NCs makes them densely packed losing in some cases their structural integrity and quantum properties.

Since the stability of MHP NCs is a prerequisite for their efficient application in devices, several approaches have been investigated to improve the stability of these NCs. One approach is to combine NCs with different classes of materials such as polymers, ${ }^{93-105}$ graphene, $^{106-111}$ 2D materials, ${ }^{112-115}$ silica ${ }^{116-120}$ and alumina. ${ }^{\mathbf{1 2 1 - 1 2 6}}$ The formation of nanocomposites through embedding LHP NCs in organic polymers as effective matrices allows a low transmission rate of oxygen and moisture. ${ }^{127,128}$ Furthermore, to avoid the chemical instability associated with $\mathrm{MAPbX}_{3} \mathrm{NCs}$, the $\mathrm{MA}^{+}$cations are replaced by $\mathrm{FA}^{+}$and $\mathrm{Cs}^{+}$. However, this imposes another form of instability which is the structural instability discussed above. As will be shown in Sections 5 and 6, the stability of MHP NCs in polar solvents and under ambient conditions can be improved through ligand modification and engineering.

\section{Perovskite nanocrystal synthesis}

Several synthetic approaches have been used and developed to produce MHP NCs. These methods can be divided into two main categories: colloidal (or non-template) and non-colloidal (or template-based) synthesis. The different synthesis routes will be discussed in this section along with the factors affecting the control over the size and shape of the NCs with the main emphasis on the impact of ligands.

\subsection{Colloidal synthesis}

Colloidal synthesis is a simple wet chemistry process where the ionic precursors are mixed under controlled temperature to form an insoluble precipitate. This well-established technique consists of different possible synthesis pathways for NCs with the two most common approaches being the hot injection (HI) and ligand-assisted reprecipitation (LARP) methods.

4.1.1 Hot injection method. The most frequently used routine for the synthesis of monodisperse colloidal NCs is the hot injection (HI) method, which was developed for the synthesis of cadmium chalcogenide NCs about three decades ago $^{129}$ and was first implemented for LHP NCs by Protesescu et al. ${ }^{57}$ in 2015. Generally, in this method, as depicted in Fig. 6a, one of the precursors is rapidly injected into a solution containing the remaining precursors and ligands that are dissolved in a high boiling point, non-coordinating solvent at high temperature. ${ }^{\mathbf{1 3 0 , 1 3 1}}$ The capping ligands are used to control the growth of the NCs in the synthesis and to passivate their surface. The nucleation starts rapidly after the injection of the precursor with a simultaneous formation of small nuclei. After
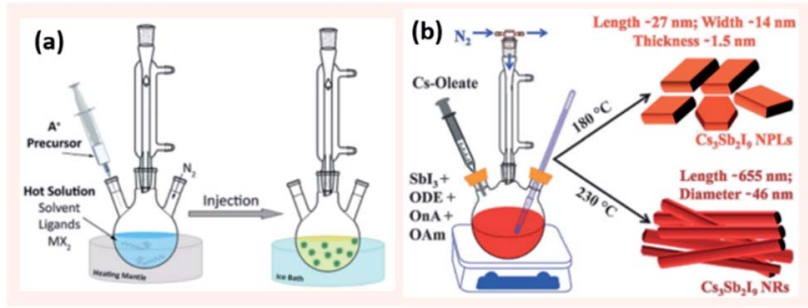

Fig. 6 (a) Schematic illustrating the HI method (b) Scheme presenting the $\mathrm{HI}$ synthesis of $\mathrm{Cs}_{3} \mathrm{Sb}_{2} \mathrm{l}_{9} \mathrm{NPLs}$ and NRs at different temperatures using octanoic acid (OnA) and oleylamine (OAm) ligands in 1-octadecene (ODE). Panels (a) and (b) are taken with permission from ref. 14 and 134 , respectively.

that, the nuclei start to grow without forming new nuclei. As the precursors become depleted, Ostwald ripening starts where the focusing regime ends and the particles size distribution slowly broadens. ${ }^{\mathbf{1} 132}$ Thus, a narrow size distribution of the NCs can be achieved in case the reaction is stopped in the size-focusing regime.

Protesescu et al. ${ }^{57}$ applied this synthesis by injecting Csoleate into a solution of $\mathrm{PbX}_{2}$ (with $\mathrm{X}$ being different halides such as $\mathrm{Cl}, \mathrm{Br}$, I) with equal ratios of $\mathrm{OA}$ and OAm ligands in the presence of octadecene (ODE) at temperatures in the range between $140{ }^{\circ} \mathrm{C}$ and $200{ }^{\circ} \mathrm{C}$ and reported the formation of monodisperse $\mathrm{CsPbX}_{3}$ nanocubes with sizes between 4 and $15 \mathrm{~nm}$. The resulting NCs were shown to have a cubic perovskite structure and a tuneable band gap through size and compositional modulation. In addition, they exhibited a narrow photoluminescence emission with full width at half maximum (FWHM) of 12-42 nm (Fig. 3a) and high PL quantum yields of 50-90\%. ${ }^{57}$ Following this report, the HI method was extended to other MHP NCs such as $\mathrm{CH}_{3} \mathrm{NH}_{3} \mathrm{PbX}_{3}\left(\mathrm{MAPbX}_{3}\right.$ with $\mathrm{X}$ being $\mathrm{Br}$ or I) by injecting methylammonium solution instead of Csoleate to the $\mathrm{PbX}_{2}$ precursor solution. ${ }^{133}$

4.1.1.1 Control of size and shape of NCs in HI method. The size, size-distribution and shape of the NCs synthesized by the HI method can be controlled by several parameters. The main factors are the temperature at which the cation or anion precursor is injected and the time at which the reaction is stopped. For example, the morphology of $\mathrm{Cs}_{3} \mathrm{Sb}_{2} \mathrm{I}_{9}$ NCs was tuned by varying the temperature where NPLs were produced at $180{ }^{\circ} \mathrm{C}$ and NRs at a higher temperature of $230{ }^{\circ} \mathrm{C}$ (Fig. 6b)..$^{\mathbf{1 3 4}}$ Due to the rapid growth of $\mathrm{CsPbX}_{3} \mathrm{NCs}$, their size was shown to be tuned mainly by the temperature rather than the growth time. ${ }^{135}$ Bekenstein et $a l .{ }^{136}$ reported the synthesis of greenemitting $\mathrm{CsPbBr}_{3}$ nanocubes at $150{ }^{\circ} \mathrm{C}$ using the HI method. At lower temperatures, anisotropic growth of the NCs proceeds favouring the growth of quasi $2 \mathrm{D}$ geometries, where cyanemitting NPLs were formed at $130{ }^{\circ} \mathrm{C}$ and very thin blueemitting NPLs were formed at $90-100{ }^{\circ} \mathrm{C}$ with lamellar structures (Fig. 7a). ${ }^{136}$ In another study, the thickness of $\mathrm{CsPbBr}_{3}$ NPLs was tuned at a monolayer level by varying the temperature and the lateral dimensions were varied by modifying the reaction time. ${ }^{137}$ In general, at higher temperatures, $\mathrm{CsPbBr}_{3}$ nanocubes are formed in the initial stage (first 10 minutes). 

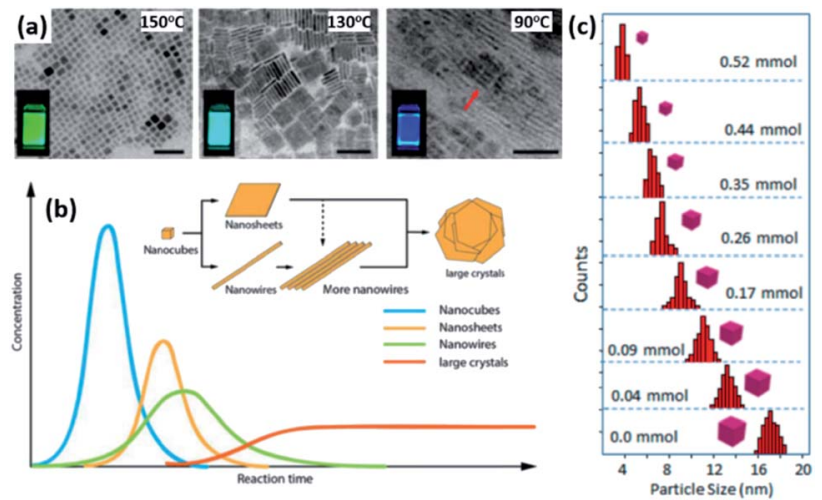

Fig. 7 (a) TEM images of $\mathrm{CsPbBr}_{3} \mathrm{NCs}$ grown at different temperatures with photos of the solutions under UV-illumination in the inset of the figures. The scale bar is $50 \mathrm{~nm}$. (b) A sketch illustrating the morphology evolution during the synthesis of $\mathrm{CsPbBr}_{3} \mathrm{NCs}$ by $\mathrm{HI}$ approach. (c) Size distribution histogram of $\mathrm{CsPbBr}_{3}$ nanocubes obtained at $160{ }^{\circ} \mathrm{C}$ from the reaction with varying amounts of oleylammonium bromide. Panels (a), (b) and (c) are adapted with permission from ref. 136, 138 and 140, respectively.

Then, NWs are formed for a reaction time up to several hours after which large crystals start to form. ${ }^{138}$ As demonstrated in Fig. $7 b$, different intermediate morphologies can coexist at a certain time. ${ }^{138}$

Another key parameter in controlling the size of the MHP NCs in HI method is the initial precursors used and their relative concentrations. Studies have demonstrated that the use of $\mathrm{ZnBr}_{2}$ or alkyl ammonium bromide as extra halide sources in the synthesis resulted in a better control of the size and size distribution of $\mathrm{CsPbBr}_{3}$ nanocubes, as shown in Fig. 7c. ${ }^{139,140}$

4.1.1.2 Role of ligands in size and shape control of NCs. Longchain organic acids and bases, mainly OA and OAm, are typically used in the HI synthesis: first to solvate the inorganic precursors in the non-coordinating solvent, then to adjust the size and shape of the synthesized NCs by modifying the reaction kinetics and lastly to stabilize the produced colloidal NCs by acting as surface ligands. ${ }^{89,141}$ A recent study on II-VI and III-V QDs has shown that the NC-ligand interaction is essential in determining the optoelectronic properties of the QDs. ${ }^{34}$ Therefore, varying the concentration of ligands, their type and chain length can significantly influence the structure, size, shape, optical properties and stability of the NCs.

It was reported that shortening the chain length of the carboxylic acid ligands used in the synthesis, without varying $\mathrm{OAm}$, increases the size of the $\mathrm{CsPBBr}_{3}$ nanocubes produced at high temperatures. Alternatively, while keeping the amount of OA fixed, the length of the amine alkyl chain was shown to affect the shape of the NCs. Nanoplatelets were formed in all cases except when using OAm at high temperature where nanocubes are produced (Fig. 8). ${ }^{141}$ From this it can be suggested that the shape selectivity depends on the chain length of the amines more than the carboxylic acids. ${ }^{141}$ Similarly, Song et al. ${ }^{142}$ demonstrated the synthesis of $1 \mu \mathrm{m} \mathrm{CsPbBr}_{3}$ NSs that are atomically thick by employing dodecylamine and $\mathrm{OA}$ in the $\mathrm{HI}$ synthesis and prolonging the reaction time.

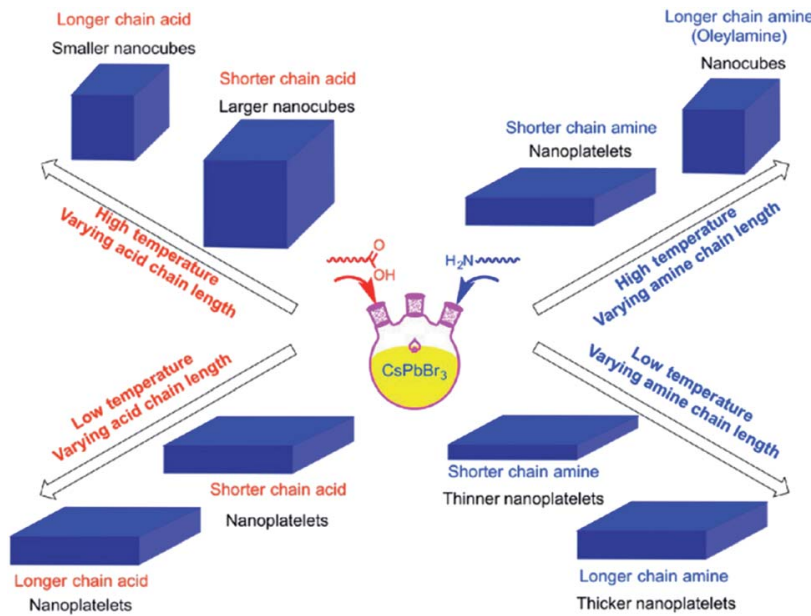

Fig. 8 Schematics illustrating the effect of temperature and type of ligand on the size and shape of NCs synthesized by the HI approach. The figure is reprinted with permission from ref. 141.

A comprehensive study was conducted by Almeida et al. ${ }^{143}$ to clarify the effect of ligand concentration on the shape, size and size distribution of $\mathrm{CsPBBr}_{3}$ NCs. They reported that reducing the concentration of $\mathrm{OA}$ and $\mathrm{OAm}$ ligands to the minimum amount required to dissolve $\mathrm{PbBr}_{2}$ suppressed the Ostwald ripening resulting in nanocubes with narrow size distribution with size ranging between 4 to $16.4 \mathrm{~nm}$. NPLs were not observed under this condition (reduced concentration of ligands) regardless of the temperature. However, increasing the concentration of both ligands enhanced the solubility of $\mathrm{PbBr}_{2}$ resulting in the formation of $0 \mathrm{D} \mathrm{Cs}_{4} \mathrm{PbBr}_{6} \mathrm{NCs}^{143}$ These results were then confirmed by Huang et al. ${ }^{144}$ who showed that the control over the size and thereby the quantum confinement of $\mathrm{MAPbBr}_{3} \mathrm{NCs}$ can be achieved by a fine control of the ligand/ precursor ratio.

Other examples include the synthesis of $\mathrm{CsPbBr}_{3} \mathrm{NWs}$ by Imran et al. ${ }^{145}$ where the width of the NWs was regulated between 10-20 nm (nonconfined) by controlling the reaction time and the octylamine/OAm ratio. Adding long alkyl chain acid (OA) with these amines resulted in the formation of NSs. However, if a carboxylic acid with a shorter alkyl chain (e.g. octanoic acid or hexanoic acid) was added to the amine ligands, thin NWs were produced with their width decreasing down to $3.4 \mathrm{~nm}$ (highly confined) when increasing the amount of short acid with respect to the amines. ${ }^{145}$ In another study, it was shown that using octylamine and octanoic acid as ligands with short alkyl chains and in addition to OA and OAm resulted in the formation of nanosheets that are a few nm thick. While the thickness stayed constant, the lateral dimensions of the nanosheets was varied from $200 \mathrm{~nm}$ to few micrometers by changing the ratio of the amount of short ligands with respect to OA and OAm. ${ }^{146}$

Furthermore, the type of capping ligand can affect the size and shape of the NCs synthesized by HI approach. For instance, Park et al. ${ }^{147}$ investigated the effect of exchanging the pristine $\mathrm{OA}$ and $\mathrm{OAm}$ ligands on $\mathrm{CsPBrr}_{3} \mathrm{NCs}$ synthesized using the $\mathrm{HI}$ 
approach with branched quaternary ammonium bromide ligands. The results showed that the NCs capped with OA/OAm or branched ligands were all monodispersed nanocubes. However, the size of the NCs increased with increasing the bulkiness of the ligands due to the lower coverage of the NC surface with the bulkier ligands, which induces rapid attachment of the remaining precursors resulting in the growth of the NCs. ${ }^{147}$ In another study, it was shown that employing branched ligands, such as triethylamine (TEAm), inhibited the formation of $\mathrm{MAPbBr}_{3}$ nanoplatelets whereas octylamine ligand facilitated the formation of NPLs. ${ }^{148}$ Studies showed that NCs capped with zwitterionic and bidentate ligands exhibit improved colloidal stability compared to monodentate ligands. ${ }^{149,150}$

4.1.2 Ligand-assisted reprecipitation method. The ligandassisted reprecipitation (LARP) method is another colloidal synthesis route that was shown to be beneficial in terms of the synthesis conditions. The LARP synthesis can be carried out in ambient conditions at RT, which makes it more cost effective than the HI method. ${ }^{151}$ In general, it includes dissolving the precursors in a solvent until an equilibrium concentration is reached, followed by moving the system into a non-equilibrium supersaturation state through decreasing the temperature, evaporating the solvent or adding a miscible solvent in which the ions have a low solubility. As a result, precipitation and crystallization occur to move the system back to equilibrium and cause the formation of NCs in the presence of ligands. ${ }^{152}$

In the case of MHP NCs, the LARP process consists of dissolving the precursor salts (typically $\mathrm{MX}_{2}$ with $\mathrm{M}$ being $\mathrm{Pb}, \mathrm{Sn}$, etc., CsX, MAX and FAX where $\mathrm{X}$ is $\mathrm{Cl}, \mathrm{Br}$ or I) in a polar solvent (as dimethylformamide (DMF), dimethylsulfoxide (DMSO)) and adding it to a nonpolar solvent (such as toluene or hexane) in the presence of ligands. Mixing the two solvents triggers

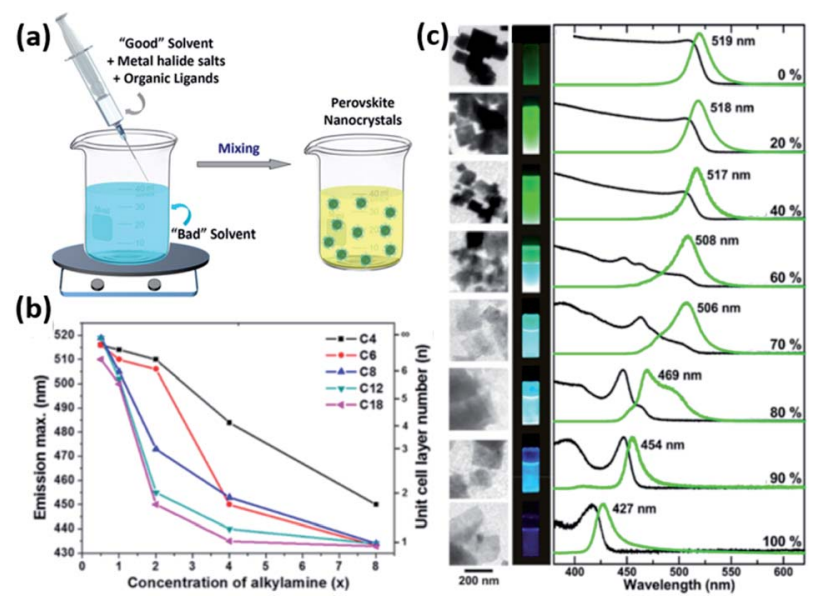

Fig. 9 (a) Illustration of the LARP synthesis. (b) The variation of PL maxima with the amount of alkylamine employed in the synthesis of $\mathrm{MAPbBr}_{3} \mathrm{NPLs}$ for different lengths of the alkyl chain of alkylamine. (c) TEM images, photographs of the NPLs under UV light, PL and UVvisible absorption spectra revealing the decrease in their thickness with the increase of octylammonium concentration (from 0 to $100 \%$ ). Panels (a), (b) and (c) are modified with permission from ref. 14, 157 and 158. immediate saturation that induces the nucleation and growth of the MHP NCs (Fig. 9a). ${ }^{151,153,154}$

Indeed, shortly before the first study on the HI synthesis of $\mathrm{CsPbX}_{3} \mathrm{NCs},{ }^{57} \mathrm{Schmidt}$ reported the LARP synthesis of highly crystalline $\mathrm{MAPbBr}_{3} \mathrm{NCs}$ in the form of $6 \mathrm{~nm}$-sized spherical nanoparticles. ${ }^{88}$ The synthesis consisted of a simple procedure where solutions of $\mathrm{MABr}$ and $\mathrm{PbBr}_{2}$ dissolved in DMF were subsequently added to a heated solution of the ligands in ODE. The formed dispersion was then precipitated by acetone. ${ }^{88}$ This technique is highly versatile, as it was extended to other MHPs forming $\mathrm{ABX}_{3}$ NCs of variable compositions ( $\mathrm{A}=\mathrm{Cs}$, MA or FA, B $=\mathrm{Pb}, \mathrm{Bi}, \mathrm{Sn}$ or $\mathrm{Sb}$ and $\mathrm{X}=\mathrm{Cl}, \mathrm{Br}$ or I) and shapes (NPLs, NWs and NRs). ${ }^{11,14,71,153,155,156}$

4.1.2.1 Role of ligands on the size and shape control of NCs. To investigate the role of ligands in the LARP approach, Zhang et al. ${ }^{58}$ conducted the synthesis of $\mathrm{MAPbBr}_{3} \mathrm{NCs}$ under different conditions by varying the ligands used in the precursor solution. The results showed that the NCs could be produced by the LARP approach when excluding the amines from the synthesis, yet control over the size was not achieved in that case. The absence of carboxylic acids in the synthesis resulted in aggregated NCs. Therefore, the amines were suggested to be used to regulate the kinetics of the crystallization and thereby the size of the NCs, whereas the acids contributed to the stability of the colloids suppressing the NCs from aggregation. ${ }^{58}$

Similar to the HI approach, it was found that higher ligand concentration in the LARP synthesis, the size of the NCs is reduced. Also, in both approaches (except at low temperatures in the HI method), the NCs become smaller, at least in one dimension, when the alkyl chain length of the ligand is increased.

For instance, the effect of ligand concentration and chain length on the morphological properties of $\mathrm{MAPbBr}_{3}$ NPLs prepared using LARP approach was inspected by Cho et al. ${ }^{157}$ The thickness of the NPLs was shown to be extremely dependent on the concentration and chain length of alkylamines (Fig. 9b). Longer amines and higher concentrations passivated the surface of the NCs more efficiently suppressing its growth in the vertical direction and allowing its growth only laterally. ${ }^{157}$ Similarly, the thickness of $\mathrm{MAPbBr}_{3}$ NPLs, produced by reacting $\mathrm{PbBr}_{2}$ with $\mathrm{MABr}$ and octylammonium bromide in the absence of carboxylic acid ligand, was shown to be proportional to the ratio of MA to octylammonium (Fig. 9c). When only octylammonium was used, "single layered" NPLs were obtained. ${ }^{\mathbf{1 5 8}}$

Moreover, it was shown that the crystal structure and composition is greatly influenced by the amount of ligands used in the LARP synthesis. Chen et al. ${ }^{159}$ revealed that by increasing the ligand content, the products changed completely from the monoclinic 3D $\mathrm{CsPbX}_{3}$ phase to the hexagonal 0D $\mathrm{Cs}_{4} \mathrm{PbX}_{6}$ phase. This was explained by the steric hindrance resulting from the increased $\mathrm{OA}$ and $\mathrm{OAm}$ in the precursor solutions making it difficult for the $\mathrm{PbX}_{6}{ }^{4-}$ octahedrons to come together and produce the 3D structure. Indeed, it was shown that OAm has a greater role in such phase transition than OA, most likely because of the attraction of $\mathrm{Cs}^{+}$to the lone-pair on the $\mathrm{N}$ atom in the amino head group of OAm resulting in more separation of the $\mathrm{PbX}_{6}{ }^{4-}$ octahedrons forming thereby the 0D phase. ${ }^{159}$ 
Other modifications to the LARP technique included the use of auxiliary ligands as benzoyl alcohol in addition to OA and octylamine. This ligand was shown to facilitate the reaction and enhance the PLQY of the produced NCs. ${ }^{148}$ Peptides with $-\mathrm{NH}_{2}$ and $-\mathrm{COOH}$ ending groups were also utilized in the LARP synthesis for a better control of the NCs' size. For instance, 12aminododecanoic acid was used as the only capping ligand in synthesizing $\mathrm{MAPbBr}_{3} \mathrm{NCs}^{\mathbf{1 6 0}}$ Using 2-adamantylammoniun bromide (ADBr) as the only capping ligand for the LARP synthesis of $\mathrm{MAPbBr}_{3}$ NCs resulted in a $100 \%$ PLQY revealing the full passivation of the surface of the NCs. ${ }^{69}$ Luo et al. ${ }^{161}$ synthesized $\mathrm{MAPbX}_{3}$ NCs using the LARP approach with replacing OA ligand with (3-aminopropyl)triethoxysilane (APTES) and polyhedral oligomeric silsesquioxane (POSS) PSS[3-(2-aminoethyl)amino]propylheptaisobutyl substituted $\left(\mathrm{NH}_{2}\right.$ POSS). These branched ligands result in the formation of smaller NCs with a better uniformity than those prepared using straight-chain ligands. The authors demonstrated the size dependence of the NCs on the amount of APTES ligand where smaller $\mathrm{MAPbX}_{3}$ nanocubes were obtained with increasing the amount of APTES due to the steric hindrance which makes the delivery of monomers to the NCs slower during the growth stage. In addition, steric hindrance and repulsion between the branched ligands increases, on one hand, the number of uncoordinated atoms at the surface of NCs lowering thereby their PLQY compared to the straight-chain ligands. On another hand, it hinders the access of solvent molecules to the NC surface, hence improving their stability compared to NCs ligated with OA. ${ }^{161}$

4.1.2.2 Emulsion LARP. An alternative approach for the synthesis of MHP NCs is through a modified LARP synthesis, termed "emulsion LARP". In this approach, as presented in Fig. 10, the precursors are dissolved in two different solvents (containing the ligands) that are immiscible, unlike the classical LARP process. When mixing the two solutions, an emulsion is formed. Upon adding a de-emulsifier that is miscible in both solvents, the reaction starts by driving the system to a supersaturation state allowing the nucleation and precipitation of the NCs. ${ }^{49,152,162,163}$ Huang et al. ${ }^{162}$ used the emulsion LARP approach to synthesize $\mathrm{MAPbBr}_{3}$ NCs by mixing the "aqueous phase" consisting of $\mathrm{MABr}$ and $\mathrm{PbBr}_{2}$ dissolved in

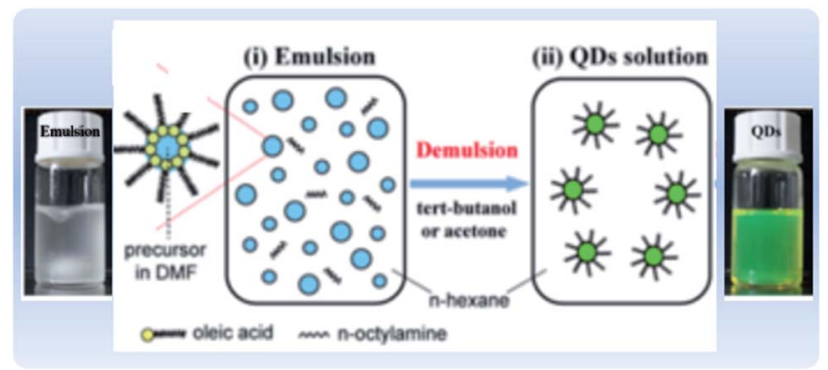

Fig. 10 Illustration of the emulsion synthesis method and photographs of the $\mathrm{MAPbBr}_{3}$ emulsion and the resultant colloidal solution upon addition of the demulsifier. Modified with permission from ref. 162.
DMF with the "oil phase" that is OA and n-octylamine in hexane. Acetone and tert-butanol could be added as de-emulsifiers to the emulsion to initiate the recrystallization and formation of the NCs. In an analogous manner, this method was used for the synthesis of $\mathrm{CsPbX}_{3}, \mathrm{MAPbX}_{3}$ and $\mathrm{FAPbBr}_{3}$ NCs with different morphologies (spherical QDs, nanocubes, NPLs and NWs). ${ }^{153,163-165}$ The method could be advantageous over conventional LARP in that it does not require that the solvents be miscible.

4.1.2.3 Polar solvent controlled ionization. In this approach, unlike the LARP method, the precursors (e.g. metal oleates and alkyl ammonium halides) are not dissociated in a polar solvent in the beginning of the synthesis. Instead, they are dispersed in a nonpolar solvent such as hexane. A polar solvent is then subsequently added to the precursors resulting in their dissociation into ions forming the NCs. Therefore, this method is advantageous over LARP strategy in using a smaller amount of polar solvents. $\mathrm{CsPbX}_{3}$ NCs synthesized by this approach exhibited PLQY over $80 \%$ and tuneable emission covering the visible range via compositional modifications. ${ }^{166}$

\subsubsection{Other colloidal synthetic approaches}

4.1.3.1 Heat-up methods. Other synthetic routes have been developed by researchers in which a mixture containing all the precursors, ligands and solvents is heated or ultrasonicated to produce the NCs under an air atmosphere and at a gram scale with high PLQY. ${ }^{68,137,167-173}$ These methods, similar to HI, required ligands based on both carboxylic acids and alkyl amines. ${ }^{14}$

The solvothermal method was demonstrated by Chen et al. ${ }^{172}$ via the synthesis of $\mathrm{CsPbX}_{3}$ NCs by heating up a mixture containing caesium carbonate or acetate and $\mathrm{PbX}_{2}$ salts with the ligands in an autoclave. The obtained NCs were in the form of nanocubes, when the precursors were heated directly in the autoclave without predissolving. However, when the precursors were dissolved prior to heating, a higher concentration of precursor ions was achieved resulting in more nuclei upon heating with the formation of $\mathrm{CsPbX}_{3}$ NWs as the result. The NWs were also obtained by prolonging the reaction time when the precursors were not predissolved. ${ }^{\mathbf{1 7 2}}$

Tong et al. ${ }^{68}$ employed a single step ultrasonication-assisted synthesis (Fig. 11a) to produce $\mathrm{CsPbX}_{3}$ NCs with a variable halide composition and morphology. They showed that $\mathrm{CsPbBr}_{3}$ nanocubes were transformed into NWs by prolonging the reaction time. ${ }^{174}$ Furthermore, modifying the initial conditions, such as the $\mathrm{Cs}_{2} \mathrm{CO}_{3}: \mathrm{PbX}_{2}$ ratio, in the ultrasonication synthesis of $\mathrm{CsPbX}_{3}$ NCs enabled tuning the fluorescence of the NCs and modifying their shape and size, as presented in Fig. $11 \mathrm{~b} .{ }^{68}$ The impact of ligands in the ultrasonication synthesis was also investigated. Employing shorter ligands (such as octylamine) instead of OAm increased the size of $\mathrm{MAPbBr}_{3}$ NCs with a wide size distribution (20-50 nm). Besides, the study revealed that increasing the amount of OAm ligand reduced the size of the NCs forming QDs as evidenced by the TEM images in Fig. 11c. ${ }^{171}$

The microwave irradiation method (Fig. 12a) was adopted and modified by several scientists for the synthesis of $\mathrm{CsPbX}_{3}$ NCs in various morphologies. ${ }^{168-170,173}$ The shape and size of NCs 


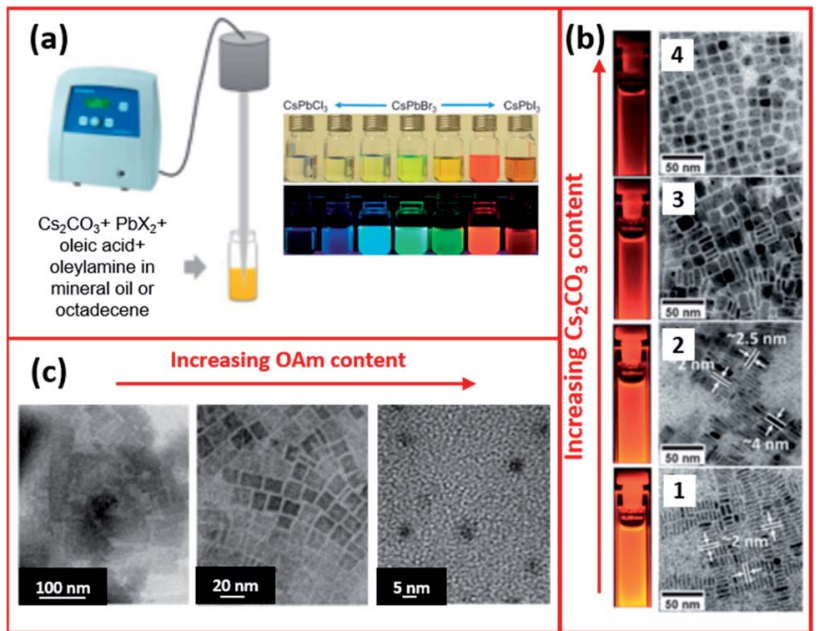

Fig. 11 (a) Schematics of the single-step tip sonication method and photograph of the synthesized $\mathrm{CsPbX}_{3} \mathrm{NCs}$. (b) TEM images (from 1 to 4) of $\mathrm{CsPb}_{3} \mathrm{NCs}_{3}$ prepared by ultrasonication method using increasing amounts of $\mathrm{Cs}_{2} \mathrm{CO}_{3}$ with respect to $\mathrm{Pbl}_{2}$. (c) TEM images showing the different morphologies of $\mathrm{MAPbBr}_{3} \mathrm{NCs}$ synthesized by the ultrasound approach with increasing amount of OAm. Panels (a) and (b) are modified with permission from ref. 68 and panel (c) from ref. 171.

synthesized using the microwave irradiation can be controlled by modifying the irradiation time and power. ${ }^{168,170}$ Long et al. ${ }^{170}$ showed that initially by increasing the irradiation time, the shape of the $\mathrm{CsPbI}_{3}$ NCs changed from being irregular nanocubes to larger regular nanocubes (Fig. 12b). The size of the nanocubes was increased with extending the irradiation time until the time where over-irradiation triggered the destruction of the $\mathrm{CsPbI}_{3}$ NCs. The power of the microwave irradiation was shown to strongly influence the PLQYs of the perovskite NCs as shown in Fig. 12c. Additionally, the ligands played a significant role in this method. For instance, changing the amount of OAm used in the synthesis modified the morphology of the NCs as seen in Fig. 12d. ${ }^{170}$
4.1.3.2 Indirect two-step synthesis from NC seeds. In addition to the aforementioned colloidal synthetic approaches, indirect two-step synthesis of MHP NCs starting from colloidal NC seeds had been demonstrated. ${ }^{175,176}$ For instance, pre-synthesized CsX NCs were used as seeds for the growth of $\mathrm{CsPbBr}_{3} \mathrm{NCs}$ by reacting with Pb-oleate. ${ }^{175}$ Similarly, 2D and 3D LHP NCs were produced by reacting $\mathrm{PbI}_{2}$ NCs with MAI or alkyl ammonium. ${ }^{176}$ This strategy is beneficial in that the easy synthesis and tuning of the parent NCs' size enables a good control of the size of MHP NCs and allows the formation of MHP NCs with complex morphologies. ${ }^{14}$

\subsection{Template-assisted synthesis}

Along with the colloidal synthesis methods, researchers have studied the growth of MHP NCs through the template-based approach. For this synthesis method, a porous matrix with nanosized open pores is used as a host for the $\mathrm{ABX}_{3}$ perovskite NCs. The infiltration of the MHP NCs within the pores can occur via three different routines. ${ }^{30}$ In the first approach, the dissolved metal halide is infiltrated then the whole scaffold is submerged in a solution containing the A cation. Therefore, the A cation penetrates the matrix and reacts with the halide forming the perovskite while the solvent evaporates from the pores. ${ }^{177}$ The second approach is to soak the porous scaffold into a mixture containing unreacted perovskite precursors. The solvent then evaporates and the perovskite NCs form in the pores after annealing. ${ }^{178,179}$ The last approach is the infiltration of a $\mathrm{NC}$ suspension, prepared $e x$ situ, into the porous template. ${ }^{180}$

In contrast to the colloidal synthesis, capping ligands are not used to prevent the aggregation of NCs in this approach. As a result, the template-assisted synthesis leads to enhanced connectivity and electrical transport between the particles which is beneficial for their application in optoelectronic devices. Moreover, since the NCs grow directly on the scaffold walls, the purification and post treatment steps are not required which are otherwise necessary for NCs synthesized by the

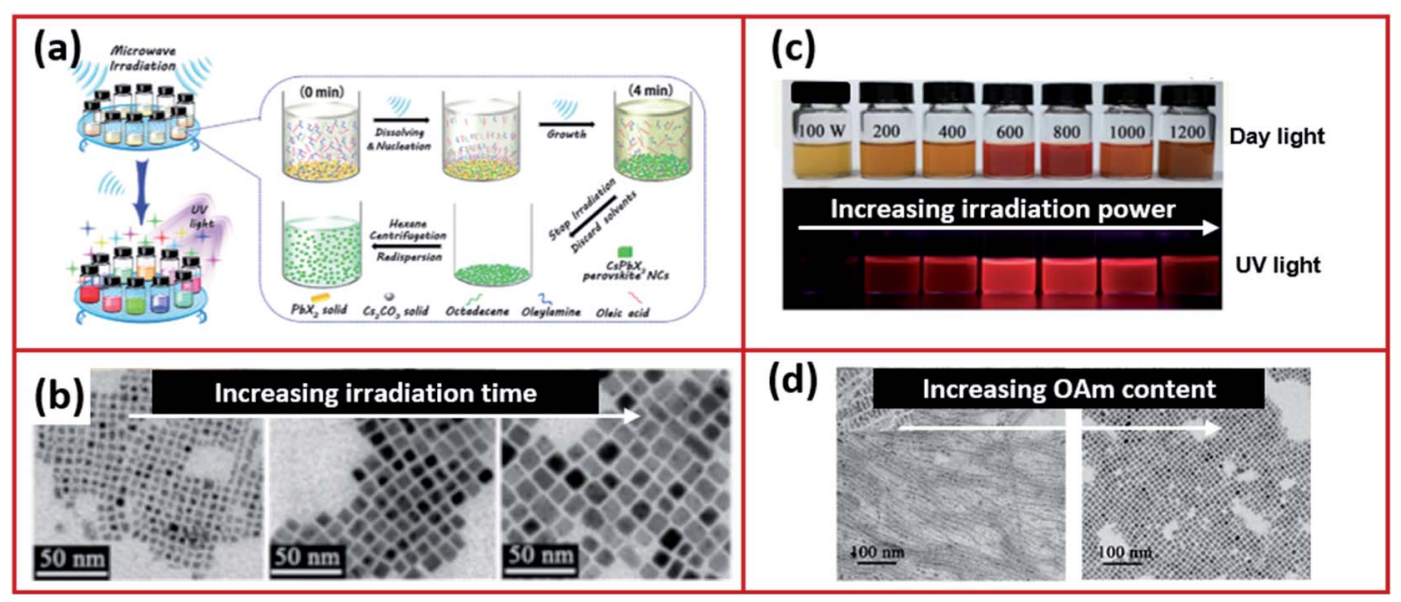

Fig. 12 (a) Illustrations of the microwave-assisted synthesis of $\mathrm{CsPbX}_{3} \mathrm{NCs}$. (b) STEM images of $\mathrm{CsPb}_{3}$ NCs with increasing microwave irradiation time. (c) A photograph of the $\mathrm{CsPb}_{3} \mathrm{NC}$ solutions, under day light and UV light, prepared by microwave irradiation at different powers. (d) TEM images showing the different morphologies of $\mathrm{CsPb}_{3} \mathrm{NCs}$ prepared by the microwave irradiation approach using increased amounts of OAm. Figure modified with permission from ref. 170. 
colloidal approaches. In this approach, the NCs are formed in a controllable environment which improves their stability and thus their performance. ${ }^{30}$ However, as a drawback to the method, the template matrix cannot be removed from the NC without damaging the materials.

To stay more focused on the review's objective in studying the impact of ligands on the properties of MHP NCs, hereafter, we will only consider the colloidal synthesis approaches in our discussions.

\section{Surface chemistry of perovskite $\mathrm{NCs}$}

For all of the above discussed colloidal synthesis approaches, a post-synthetic purification step is typically required. In the purification procedure, the NCs are isolated and the excess surfactants and precursors or byproducts are removed, which is necessary before integrating the produced NCs in devices. The purified NCs are obtained through several steps of precipitation using a polar/nonpolar solvent pair followed by centrifugation and redispersion. The purification of the perovskite NCs is critical as they can dissolve and degrade or agglomerate by losing their ligands upon repetitive washing steps with polar solvents reducing thereby their PLQY and colloidal stability. ${ }^{33,162,181}$ To overcome this challenge, it is essential to thoroughly understand the surface chemistry of the perovskite NCs by investigating their surface termination and the bonding between the NCs and the ligands.

Furthermore, the surface chemistry of a material is known to be a key factor that influences its interactions and properties and thereby its application. The following section will highlight the research outcomes and understandings achieved regarding the surface chemistry and ligation in MHP NCs.

\subsection{Surface-ligand interaction}

5.1.1 Nature of surface ligation in inorganic perovskites. Different tools have been utilized to investigate the interface between the NCs and the capping ligands such as: Fourier transform infrared spectroscopy (FTIR), Nuclear Magnetic Resonance (NMR) spectroscopy and X-ray photoelectron spectroscopy (XPS). ${ }^{1} \mathrm{H}$ NMR combined with the two modified NMR techniques, nuclear Overhauser effect spectroscopy (NOESY) and Diffusion Ordered NMR Spectroscopy (DOSY), are powerful tools that can be used not only for structural analysis of the organic components but also to probe the dynamics of the ligand bonding to the NC surface. ${ }^{33,182}$ NOESY and DOSY are used to assess the ligation as they allow the differentiation between free and bound ligands. ${ }^{33,94}$

So far, most of the surface investigations have focused on lead halide perovskite NCs; and specifically on $\mathrm{CsPbX}_{3} \mathrm{NCs}_{\text {. In }}$ general, it was found that these NCs are stabilized by a dynamic interaction between the ligands, that are usually an ionic pair, and the oppositely charged ions at the NCs surface. The anion of this ionic pair is typically alkyl carboxylate or a halide ion and the cation is $\mathrm{Cs}^{+}$or alkyl ammonium. ${ }^{\mathbf{1 8 3}}$ Furthermore, the processing procedures (such as the purification steps and solvents used) were shown to affect the ligand composition on the NC surface. This depends on the strength of ligands binding to the NC surface, where, for instance, the oleate binds more strongly to the NC surface compared to ammonium.

De Roo et al. ${ }^{33}$ reported one of the first studies on the surface ligation in inorganic perovskite NCs. In their study, they investigated the surface of $\mathrm{CsPBBr}_{3} \mathrm{NCs}$, synthesized by the HI approach, after purification with acetone and redispersion in hexane. The HI synthesis of $\mathrm{CsPbX}_{3}$ NCs by reacting Cs-oleate and $\mathrm{PbX}_{2}$ yields $\mathrm{Pb}$-oleate and oleylammonium bromide (OAmH-Br) byproducts, both of which can act as binding species to the NC surface along with the OA and OAm. However, due to the highly ionic character of $\mathrm{CsPbBr}_{3} \mathrm{NCs}$, OAmH-Br is preferably bound to the NCs compared to Pb-oleate. Concerning OA and OAm ligands, ${ }^{1} \mathrm{H}$ NMR spectra revealed that OA was not bound to the NC surface and OAm was present as the oleylammonium ion $\left(\mathrm{OAmH}^{+}\right)$. Therefore, based on the NMR analysis and these ligand possibilities, it was suggested that $\mathrm{OAmH}$ $\mathrm{Br}$ acts as an X-type ligand binding to the surface of the acetonepurified NC sample, where the bromide anion binds to surface $\mathrm{Cs}^{+}$and $\mathrm{Pb}^{2+}$ and the $\mathrm{OAmH}^{+}$cation binds to $\mathrm{Br}^{-}$on the $\mathrm{NC}$ surface. ${ }^{33}$ Using DOSY measurements, the diffusion coefficient of OAmH-Br was found to be an average of diffusion coefficients of free and bound $\mathrm{OAmH}-\mathrm{Br}$, with the bound fraction being $65 \%$ corresponding to almost complete passivation of the surface and a ligand density of $2.9 \mathrm{~nm}^{-2}$. The surface-ligand interaction in this acetone-purified $\mathrm{CsPbX}_{3}$ NC sample is schematized in Fig. $13 \mathrm{a}^{33}$

In the same work, De Roo et al. ${ }^{33}$ investigated the role of amines by adding excess OAm to the acetone-purified $\mathrm{CsPbBr}_{3}$ NCs. The results showed that OAm is involved in several equilibria (as shown in Fig. 13b): (a) it undergoes acid/base equilibrium reaction with $\mathrm{HBr}$ producing $\mathrm{OAmH}-\mathrm{Br}$ that binds as $\mathrm{X}$ type ligand to the NC surface, (b) it deprotonates OA yielding oleylammonium oleate that is also an X-type ligand with the oleate and $\mathrm{OAmH}^{+}$binding to the NC surface as an ion pair and (c) it binds in its deprotonated form (OAm) to the surface cations as an L-type ligand. For the last two cases, a ligand exchange occurs between the initial OAmH-Br bound to the surface and the oleylammonium oleate or OAm. This is because the NC surface was fully passivated before the addition of OAm,

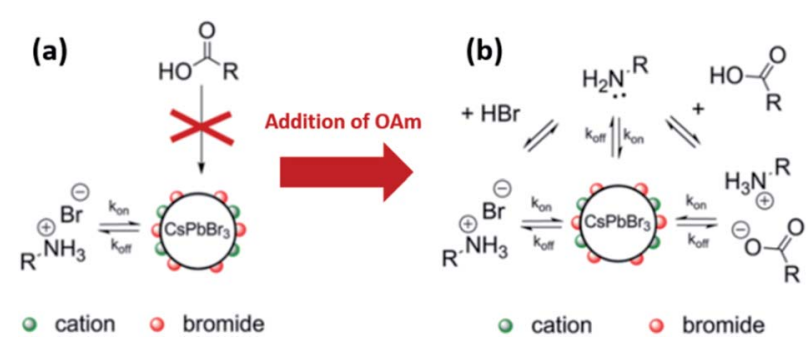

Fig. 13 Schematic representations of the dynamic surface stabilization of $\mathrm{CsPbBr}_{3} \mathrm{NCs}$ purified with acetone and dispersed in hexane (a) by oleylammonium bromide before the addition of excess OAm and (b) by oleylammonium bromide, oleylammonium oleate and OAm after the addition of excess OAm. The figure is modified with permission from ref. 33 . 
as discussed before. Measurements based on the different NMR techniques indicated that the oleylammonium oleate ligand was tightly bound to the NC surface. ${ }^{33}$

Hence, the as-synthesized $\mathrm{CsPbBr}_{3}$ NCs are stabilized by the dynamic interaction with $\mathrm{OAmH}-\mathrm{Br}$, which exchanges between its free and bound states. With the addition of amine, oleate binds tightly to the NC surface as an ion pair with the amine and is influenced by the existing acid/base equilibria in the NC solution. Based on these findings, an improved purification protocol of the $\mathrm{CsPbBr}_{3}$ NCs was demonstrated in which both (the acid and the amine) are added with an excess of amine to the NC solution before the precipitation with acetone. This was shown to be beneficial for maintaining the colloidal stability and PLQY with several precipitation/redispersion steps due to the tightly bound oleylammonium oleate. ${ }^{33}$

The labile nature of alkyl ammonium bonding to the NC surface was also revealed by Pan et al. ${ }^{141}$ Using ${ }^{1} \mathrm{H}$ NMR and FTIR spectroscopy, it was shown that both alkyl ammonium and carboxylate bind to the surface of $\mathrm{CsPBBr}_{3}$ NCs in toluene or hexane with the alkyl ammonium being more susceptible to detachment from the NC surface during purification and washing with polar solvent. The work was based on replacing $\mathrm{Cs}_{2} \mathrm{CO}_{3}$ in the NC synthesis with caesium acetate, which permits using short and saturated alkyl chain acids and bases that have low boiling points. This enables a better assessment of the surface ligands because the overlapping resonances of ODE, alkyl ammonium and oleate in the NMR spectra can then be separated. NMR and FTIR measurements indicated that alkyl ammonium was significantly reduced when the NCs were washed with hexane/acetone, yet it was retained when washing with only hexane. For both washing procedures, it was shown that the carboxylate was not removed. These results were reproducible for a variety of acid-base combinations with different alkyl chain lengths. Based on that, the solvent dependence of the surface ligands was attributed to a weaker ammonium-surface interaction in comparison to carboxylatesurface bonding, which is consistent with the findings of De Roo et $a l^{33}$ The metal-ligand coordination between the carboxylates and the surface $\mathrm{Pb}$ atoms is stronger and more robust towards polar solvent wash than the hydrogen bonding interaction between the surface bromide and the ammonium..$^{141}$

5.1.2 Effect of surface ligation on stability of inorganic perovskite NCs. The impact of purification on the ligation in $\mathrm{CsPbX}_{3} \mathrm{NCs}$ and consequently its effect on their instability was investigated by Grisorio et al. ${ }^{182}$ They showed that the origin of instability in the as-prepared NCs is the formation of amides from the condensation of carboxylic acid and amine ligands. Before purification, several equilibria occur on the NC surface involving the remaining unreacted precursors (Cs-oleate), the excess OA and OAm ligands (Fig. 14a). The passivation with $\mathrm{OAmH}^{+}$involves the release of a proton on the NC surface. The adsorbed protons and the unwashed Lewis acid species make the NC surface acidic, which catalyzes the formation of amide from the OA and OAm ligands (Fig. 14b)..$^{182}$ The amide formation in perovskite NCs was also reported in other studies. ${ }^{33,125,184}$ Upon condensation into an amide, the coordinating groups for
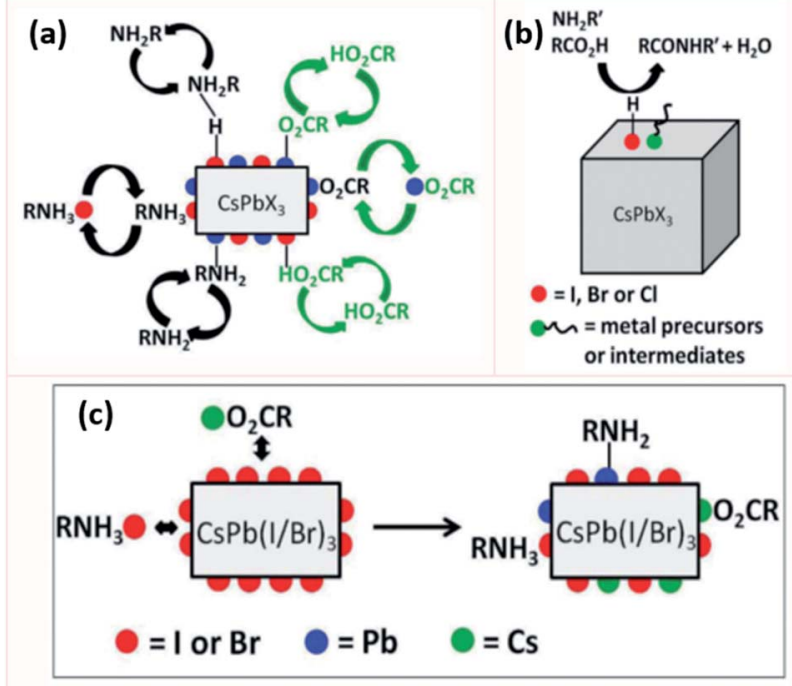

Fig. 14 Schematic representation showing in (a) the equilibria of all the ligands involved in the passivation of $\mathrm{CsPbX}_{3} \mathrm{NCs}$, (b) the amide formation by the adsorbed acidic species on the NC surface and in (c) the modification of the surface of $\mathrm{CsPb}(\mathrm{l} / \mathrm{Br})_{3} \mathrm{NCs}$ upon purification. This figure is modified with permission from ref. 182.

the ligands become unavailable to bind to the NCs surface, hence destabilizing the NCs.

By investigating the surface of $\mathrm{CsPbX}_{3} \mathrm{NCs}$ in non-polar solvents using NMR spectroscopy, it was shown that oleate strongly binds to undercoordinated $\mathrm{Pb}$ and $\mathrm{Cs}$ atoms, OAm weakly binds to surface protons and uncoordinated $\mathrm{Pb}$, and carboxylic acid exchanges with tightly bound oleate. The equilibrium between these species shifts in polar solvents favoring the desorption of oleylammonium halide from the NC surface. During purification with polar antisolvent, the adsorbed acidic species are removed from the NC surface inhibiting the degradation of the organic shell through the formation of amide, which improves the stability of the NCs. ${ }^{182}$

${ }^{1} \mathrm{H}$ NMR spectroscopy revealed that $\mathrm{CsPbCl}_{3}$ and $\mathrm{CsPbBr}_{3}$ NCs have very similar surface chemistry; while more amides were observed with $\mathrm{CsPbI}_{3}$ NCs. This different behavior is attributed to the soft character of I atoms which favors the interaction with OA. Consequently, OA strongly binds to the

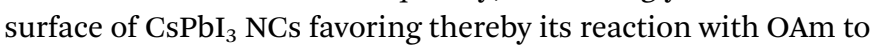
yield the amide. As a result, when exposed to polar solvents or moisture, the surface will undergo structural decomposition as most of the ligands are lost that explains its lower stability compared to $\mathrm{CsPbBr}_{3}$ and $\mathrm{CsPbCl}_{3} \mathrm{NCs}^{182}$ The instability of the iodide-based perovskite NCs is also attributed to the weaker acid-base interaction between oleylammonium ligand and $\mathrm{I}^{-}$ compared to $\mathrm{Br}^{-}$which makes them more susceptible to the loss of ligands during the purification procedure..$^{53}$

The Cs-enriched surface of $\mathrm{CsPbI}_{3}$ is passivated by the labile Cs-oleate and oleylammonium iodide that compete in both non-polar and polar solvents. Yet, in the case of $\mathrm{CsPbBr}_{3}$ and $\mathrm{CsPbCl}_{3}$, the stronger lead-halide bonds would hamper the dissociation of ammonium in non-polar solvents. In addition, 
they would allow a stronger interaction of the surface $\mathrm{Cs}^{+}$with oleate. In the case of the unpurified $\mathrm{CsPb}(\mathrm{Br} / \mathrm{I})_{3} \mathrm{NCs}$, an I-rich surface is exposed since it is more difficult for the larger $\mathrm{I}^{-}$to incorporate into the crystal lattice compared to $\mathrm{Br}^{-}$. As a result, these mixed NCs exhibit similar ligation to $\mathrm{CsPbI}_{3}$ showing therefore a large amount of amide and simultaneous presence of labile oleylammonium and Cs-oleate. Purifying these mixed perovskite NCs removes the dangling iodides, thus exposing strongly bound $\mathrm{Cs}$ and under-coordinated $\mathrm{Pb}$ atoms that are then more efficiently passivated by OA and OAm (Fig. 14c). Hence, these NCs show improved stability after purification. ${ }^{\mathbf{1 8 2}}$

\subsection{Perovskite NC surface termination}

Understanding the surface termination of the NCs is essential as diverse surface terminations are expected to result in different optical and electronic properties. ${ }^{\mathbf{1 8 5}}$ The termination of MHP NCs of the general formula $\mathrm{ABX}_{3}$ could be an $\mathrm{AX}^{\mathbf{1 8 5 , 1 8 6}}$ or $\mathrm{BX}_{2}$ (ref. 36 and 185) rich surface. $\mathrm{CsSnI}_{3}$ terminated with CsI, for example, have shown a higher electron mobility than $\mathrm{CsSnI}_{3}$ terminated with $\mathrm{SnI}_{2}{ }^{187}$ Combining FTIR, X-ray fluorescence and XPS techniques, Leng et al. ${ }^{188}$ showed that the surface of $\mathrm{MA}_{3} \mathrm{Bi}_{2} \mathrm{Br}_{9}$ QDs is Br-rich and that the surface $\mathrm{MA}^{+}$is replaced with the protonated octylamine ligand, while the OA was found to be bound to Bi ions. The surface of $\mathrm{MAPbBr}_{3}$ NCs was suggested to be surface terminated with $\mathrm{MA}^{+}$and $\mathrm{Br}^{-}$with the $\mathrm{Br}$ : $\mathrm{Pb}$ molar ratio being 3.55. ${ }^{58}$ Similarly, for $\mathrm{CsPbBr}_{3} \mathrm{NCs}$, the $\mathrm{Br}: \mathrm{Pb}$ ratio was found to be 3.2 (ref. 189) and the surface was enriched with $\mathrm{Cs}^{+}$and $\mathrm{Br}^{-}$despite the excess $\mathrm{PbBr}_{2}$ used in the synthesis. ${ }^{185}$ However, another study by Maes et al. ${ }^{190}$ suggested that the surface $\mathrm{Cs}^{+}$ions in $\mathrm{CsPbBr}_{3}$ NCs are substituted with $\mathrm{OAmH}^{+}$and that the surface is partially terminated with $\mathrm{PbBr}_{2}$ since the ratio of $\mathrm{Cs}$ to $\mathrm{Pb}$ was lower than 1 particularly in small NCs.

Elemental analysis of $\mathrm{CsPbX}_{3} \mathrm{NCs}$ via energy dispersive X-ray spectroscopy (EDX) coupled with field emission gun scanning electron microscopy (FEG-SEM) revealed that these NCs have a Cs-enriched surface. The exposed Cs atoms at the NC surface can be then replaced by oleylammonium ions. Hence, the surface of $\mathrm{CsPbX}_{3}$ NCs contains $\mathrm{Cs}^{+}$, oleylammonium ions and halides. ${ }^{182}$ These results are analogous to the findings of Ravi et $a l .{ }^{191}$ in which an investigation of the $\mathrm{CsPbBr}_{3}$ NCs composition using synchrotron-based XPS revealed that they have a CsBr surface termination. Using ${ }^{1} \mathrm{H}$ NMR measurements and first-principles DFT (density functional theory) calculations, they also showed that the substitution of $\mathrm{Cs}^{+}$with $\mathrm{OAmH}^{+}$is energetically feasible and stabilizes the NCs through the formation of hydrogen bonds between $-\mathrm{NH}_{3}{ }^{+}$group and the $\mathrm{Br}^{-}$ ions on the surface. Indeed, as presented in Fig. 15a, there are three $\mathrm{H}$ bonds: two $\mathrm{H}$ bonds with $\mathrm{Br}^{-}$on the surface and a weaker $\mathrm{H}$ bond with $\mathrm{Br}^{-}$in the underneath layer. ${ }^{191}$ This study also confirmed the outcomes of De Roo's work ${ }^{33}$ in that OA is not bound to the NC surface and that a constant ratio of OAm/ $\mathrm{OA}$ or their protonated/deprotonated forms $\left(\mathrm{OAmH}^{+}\right.$and oleate) must be maintained to stabilize the NC solution. Indeed, a ratio of OAm : OA $=1: 5$ resulted in the best PLQY and stability through maintaining this acid/base equilibrium. ${ }^{191}$

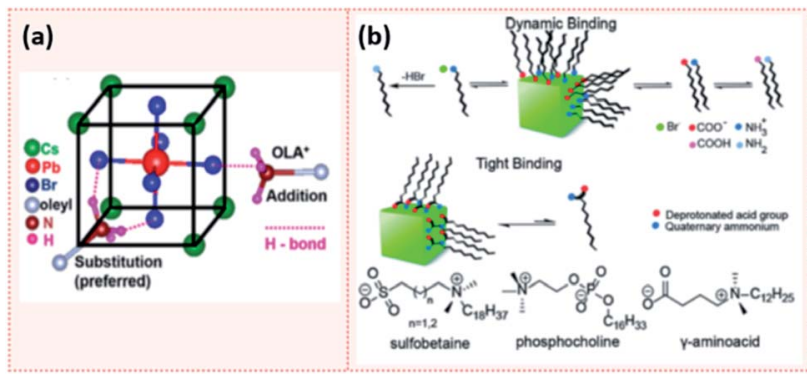

Fig. 15 (a) Schematic showing the hydrogen bonding between oleylammonium $\left(\mathrm{OLA}^{+}\right)$and $\mathrm{Br}^{-}$on the NC surface. The addition of OLA to the surface results in $1 \mathrm{H}$ bond while the substitution of $\mathrm{Cs}^{+}$with $\mathrm{OLA}^{+}$which is the more preferred results in $3 \mathrm{H}$ bonds. (b) A scheme showing the dynamic binding of conventional acid and amine ligands and the tight binding of zwitterionic ligands to the perovskite NC surface. The molecular structure of some examples of zwitterionic molecules are also presented. Panels (a) and (b) are taken with permission from ref. 191 and 149.

Additional studies revealed that the presence of $\mathrm{Br}$ vacancies on the surface results in under-coordinated $\mathrm{Pb}$ atoms that act as trapping sites reducing the PLQY. The addition of excess $\mathrm{PbBr}_{2}$ in a solution of carboxylic acid and amine introduces excess $\mathrm{Br}^{-}$ and results in a stronger ligand binding to the surface which improves the surface passivation and thereby the PLQY. ${ }^{\mathbf{1 9 2}}$ The under-coordinated $\mathrm{Pb}^{2+}$ ions can be passivated with thiocynate salts (e.g. $\mathrm{NH}_{4} \mathrm{SCN}$ or NaSCN), for instance, to effectively reduce the nonradiative carrier recombination pathways and leading to near-unity PLQY.9,72

\subsection{Purification and isolation of NCs}

Research groups have reported different strategies for the purification and isolation of the NCs with a variety of antisolvents and centrifugation parameters based on the composition and morphology of the NCs..$^{57,136,138,162,193-195}$ Due to the weak and dynamic bonding between the NC surface and the ligands, ${ }^{33,34}$ the purification process can result in a decline in the photoelectric properties and stability of the NCs and even in some cases loss of their structural integrity due to the desorption of ligands from the surface by the polar solvents used. ${ }^{33}$ This is especially relevant in the case of iodide-based perovskite NCs which are more susceptible to the loss of ligands during the purification. As a result, the NCs would agglomerate or convert into the photo-inactive phase. ${ }^{53}$

One strategy was demonstrated by the work of De Roo et al. ${ }^{33}$ as discussed above, where excess OA and OAm were added for the purification of $\mathrm{CsPbBr}_{3}$ NCs to manipulate the bonding equilibrium between the bound and free ligands. The same purification procedure was adapted by Grisorio et al. ${ }^{\mathbf{1 8 2}}$ on $\mathrm{CsPbX}_{3}$ NCs with different halide compositions. The authors showed that the excess OA and OAm during purification was beneficial in preserving the colloidal integrity of the NCs for all halide compositions except for $\mathrm{X}=\mathrm{I}$, where the NCs undergo a phase transition as soon as they contact the polar solvent. However, repeating the washing steps resulted in the loss of structural integrity of the NCs due to desorption of ligands. 
Therefore, the successful purification protocol preserving the stability of $\mathrm{CsPbX}_{3}$ NCs (except $\mathrm{CsPbI}_{3}$ ) consisted of a single step, where the products from centrifugation were dispersed in hexane with excess OA and OAm added. The NCs are then precipitated with an equal volume of acetone and recollected from a second centrifugation. ${ }^{182}$

Swarnkar et $a .^{53}$ proposed a method for purifying $\mathrm{CsPbI}_{3}$ NCs, after examining several low polarity antisolvents. In this method, methyl acetate (MeOAc) was used as an antisolvent to remove the unreacted precursors and isolate $\mathrm{CsPbI}_{3} \mathrm{NCs}$ without full removal of surface species; thereby preventing the NCs agglomeration. MeOAc was chosen because it has both the needed polarity for precipitating the NCs and the low solubility preventing the removal of surface ions. The purified NCs, dispersed in hexane, were shown to be stable in the cubic phase for months in ambient conditions compared to only several days for the unpurified NCs. However, an excess of MeOAc caused the destabilization of the NCs and their aggregation due to the removal of the capping ligands. ${ }^{53}$

Another attempt for purifying perovskite NCs was proposed by Krieg et al. ${ }^{\mathbf{1 4 9}}$ via employing 3-(N,N-dimethyloctadecylammonio)propanesulfonate as a long chain zwitterionic ligand. Using this ligand, $\mathrm{CsPbBr}_{3}$ NCs were isolated after four precipitation/redispersion rounds with a preserved high PLQY (above 90\%). The authors suggested that the use of commercially available zwitterionic molecules (e.g. phosphocholines, $\gamma$ amino acids and sulfobetaines) as capping ligands offers two main advantages over the carboxylic acid and amine ligands (Fig. 15b). First, their cationic and anionic groups are not involved in acid-base equilibria. This makes them strongly attached to the NC surface, unlike the highly dynamic bonding between the carboxylate and ammonium moieties with the NC surface caused by the acid-base interactions. Furthermore, the binding of these ligands to the surface is stabilized by chelation. ${ }^{149}$ Hence, the use of zwitterionic ligands is considered to be a promising strategy for maintaining the colloidal stability during the purification process. This is due to the stronger bonding between the ligands and the NCs, compared to the conventional acid and amine ligands, which inhibits the desorption of the protective ligand shell.

\section{Surface manipulation for use of perovskite NC films}

Numerous studies on employing MHP NCs in lasers, photodetectors, electroluminescent devices, LEDs and solar cells have been reported. ${ }^{\mathbf{9 1 3 , 1 9 6 , 1 9 7}}$ For such applications, thin films based on the colloidal NCs have to be fabricated, as suspensions of MHP NC are not practical for use in devices since concentrations and compositions can change with time. Furthermore, for optoelectronic applications it is impractical to make electrical contact to solutions since the faradaic efficiency will be low. Suspended in solution, NCs are dilute and only weakly interacting with each other, which minimizes interfacial reactions and electronic communication between NC. Thin films can be prepared from NC solutions by spin coating, self-assembly and
3D printing for use in large area and flexible devices. Yet, the deposition of these highly luminescent NCs into solid-state films has resulted in a reduction in their PLQY, which negatively affects their use in optoelectronic applications. ${ }^{198}$ Similarly, the use of porous matrices to grow MHP NCs for applications reduces the optical quality due to aggregation of the host particles and their large size. To successfully employ perovskite NCs in solid state devices, different strategies involving ligands and therefore surface manipulation of have been used, which we will summarize below: (1) ligand exchange, (2) using short, bidentate or branched ligands in the NC synthesis and (3) controlling the ligand density on the NC surface.

\subsection{Ligand exchange}

The long chain ligands that are typically used in the synthesis of MHP NCs are electronically insulating, which impedes charge carrier injection and transport at the interfaces in optoelectronic devices. Furthermore, the labile nature of ligand bonding to the NC surface reduces the stability and the photoluminescence of the NCs during the purification and processing steps. Therefore, for efficiently introducing MHP NCs into optoelectronic devices, these insulating weakly bound long chain molecules must be exchanged with shorter ones that allow better charge transfer or with ligands that have a stronger bonding to the NC surface leading to a better stability. This can be established through ligand exchange in solution or a solidstate ligand exchange.

6.1.1 Solution-based ligand exchange. In solution-based ligand exchange, the capping ligands at the surface of MHP NCs are replaced with new ligands in the colloidal solution prior to the deposition of the NC films. This postsynthetic approach is mostly used to replace the long OA and OAm ligands in NCs to enable their utilization in applications. Several ligands have been used for ligand exchange in solution and the influence of the alkyl chain length and bulkiness of these ligands on the optoelectronic properties and stability of the resulting NCs has been investigated. ${ }^{\mathbf{1 4 7}, \mathbf{1 9 9}, 200}$

The solution-based ligand exchange is usually done through a two-step process, in which the first step includes the addition of OA to protonate OAm. The protonated OAm reacts with the deprotonated OA forming an acid/base complex that desorbs from the surface enabling the subsequent adsorption of the excess OA on the QD surface. Following this step, a ligand, typically an X-type ligand, is introduced into the NC solution, which fully replaces the OA and remaining OAm on the NC surface.

An example of such a ligand exchange was demonstrated by Pan et al. ${ }^{199}$ where di-dodecyl dimethyl ammonium bromide (DDAB) ligand was used to replace OA and OAm ligands on the surface of $\mathrm{CsPbX}_{3}$ QDs synthesized by the HI approach (Fig. 16a). This exchange occurs because the $\mathrm{Br}^{-}$ions of DDAB have a stronger affinity than the oleate group to the surface $\mathrm{Pb}^{2+}$ or $\mathrm{Cs}^{+}$ions. The intermediate step of OA addition was shown to be essential as the direct addition of DDAB to the QDs caused their degradation. ${ }^{199}$ As observed in Fig. 16b, the ligand 

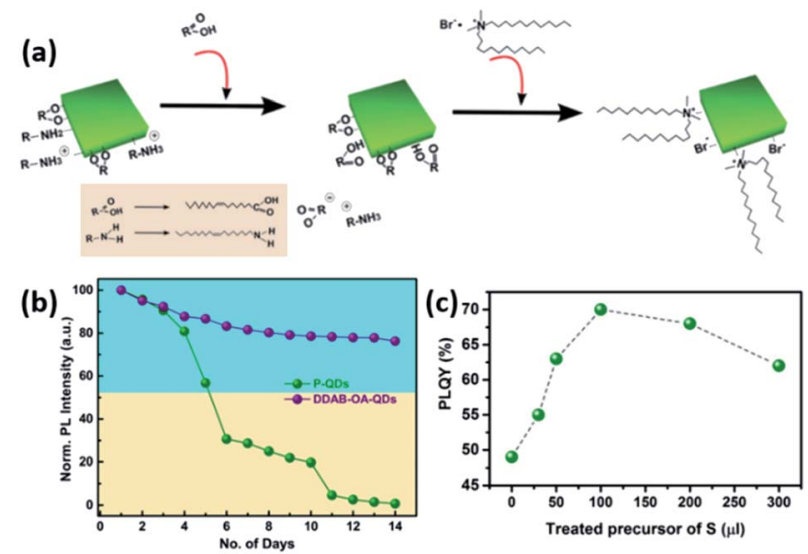

Fig. 16 (a) Illustration of the ligand exchange mechanism on $\mathrm{CsPbBr}_{3}$ QDs. (b) The variation of $\mathrm{PL}$ intensity with respect to time for unmodified QDs and DDAB-OA treated QDs. Both (a) and (b) are taken with permission from ref. 199. (c) The variation of PLQY values of $\mathrm{CsPbBr}_{3} \mathrm{QDs}$ with the amount of the sulfide precursor added. This panel is taken with permission from ref. 200.

exchanged QD solution showed better stability than the untreated one due to the large steric hindrance of the $\mathrm{DDA}^{+}$ions adsorbed on the QD surface. The application of DDAB-treated $\mathrm{CsPbBr}_{3}$ QDs in LED devices resulted in external quantum efficiency (EQE) of 3\%, which was much higher than that of the untreated QDs. This was attributed to the improved charge carrier balance in the QD films treated with this halide-ion pair ligand and the enhanced charge transport due to better crystallinity and less defects in the DDAB-treated QD films. ${ }^{199}$

A similar hybrid organic-inorganic ion pair, di-dodecyl dimethyl ammonium sulfide (DDAS), was employed to passivate the surface of $\mathrm{CsPbBr}_{3}$ QDs using the same approach. ${ }^{200}$ Elemental analysis of the samples revealed that both $\mathrm{DDA}^{+}$and $\mathrm{S}^{2-}$ play a role in the passivation. The treatment of the QDs with this sulfur precursor improved the PLQY significantly from $49 \%$ to $70 \%$ (Fig. 16c). It also endowed the QDs with superior stability in ambient conditions with $\sim 60 \%$ humidity overcoming one of the major issues that hinder the application of MHP NCs. Films based on the treated QDS showed considerable stability under continuous pulsed laser irradiation for 34 hours, which enabled inducing ultrastable amplified spontaneous emission (ASE) in these films. ${ }^{200}$

By replacing OAm in $\mathrm{CsPbBr}_{3}$ NCs with quaternary ammonium bromide (QAB) ligands of different chain lengths and bulkiness to the OA and OAm, Park et al. ${ }^{147}$ showed that the NCs surface was better passivated with the less bulky ligands resulting in improved PLQY and stability of the NCs. The chain length did not have a considerable effect on the optical properties of the NCs, yet the charge carrier transport in films based on these NCs was enhanced with the decrease of the ligand chain length. LED devices fabricated using the NC films with optimized ligand chain length and bulkiness achieved high $\mathrm{EQE}$ of $9.71 \%$ that is 16-fold enhancement compared to $\mathrm{OA} /$ OAm-capped $\mathrm{CsPbBr}_{3} \mathrm{NCs}{ }^{\mathbf{1 4 7}}$

Ye et $a l .{ }^{201}$ investigated an opposite ligand exchange strategy where two short chain ligands (propionic acid and butylamine) anchored to $\mathrm{CsPbBr}_{3}$ NCs were replaced with longer chain ligands. The ligand exchange was done by adding long chain acid and amine ligands to the NC solution prior to purification. The spincoated NC films became more uniform after the ligand exchange and resulted in improved LED performance. Even though the shorter chain ligands lead to more efficient charge transport properties, the NCs capped with the long chain OA/ OAm pair achieved the highest LED performance and best thermal stability because of better defect passivation. ${ }^{\mathbf{2 0 1}}$

In addition to the modification of the NC surface, phase transformation, that is usually combined with shape evolution, can occur when adding new ligands to a solution of the presynthesized NCs. For instance, the addition of amines to $\mathrm{CsPbBr}_{3} \mathrm{NCs}$ leads to a 3D to 0D transformation to $\mathrm{Cs}_{4} \mathrm{PbBr}_{6}$ NCs through the extraction of $\mathrm{PbBr}_{2}$ from the 3D structure and its complexation with the amines. The same process occurs on $\mathrm{CsPbBr}_{3} \mathrm{NC}$ films exposed to volatile butylamine vapour where they transform into the $0 \mathrm{D} \mathrm{Cs}_{4} \mathrm{PbBr}_{6}$ NCs and back to the 3D $\mathrm{CsPbBr}_{3}$ upon evaporation of butylamine. ${ }^{202}$ This 3D to 0D phase transformation was also reported by Liu et al. ${ }^{203}$ via the addition of OAm with various alkyl thiol ligands to $\mathrm{CsPbBr}_{3}$ NCs. Similarly, the ligand-assisted phase transformation of 3D $\mathrm{CsPbBr}_{3}$ NCs into 2D $\mathrm{CsPb}_{2} \mathrm{Br}_{5}$ NSs was realized through the addition of DDAB to the NC colloidal solution. The transformation occurred first through exfoliation where $\mathrm{Pb}^{2+}$ complexes with $\mathrm{Br}^{-}$from DDAB forming $\left[\mathrm{PbBr}_{3}\right]^{-}$and $\left[\mathrm{Pb}_{2} \mathrm{Br}_{5}\right]^{-}$ complexes followed by a reorganization step to form the $\mathrm{CsPb}_{2} \mathrm{Br}_{5} \mathrm{NSs}^{204}$

6.1.2 Solid-state ligand exchange. Solid-state ligand exchange, which is the conventional method used in metal chalcogenide QDs, is based on dropping a solution of the new ligand dissolved in an antisolvent for the QDs onto the QD film. However, applying this method to perovskite NCs is challenging because of their poor stability in the polar antisolvents used in this process. Hence, for solid-state ligand exchange, the solubility of the ligands and the NCs capped with the new ligands must be controlled by selecting the appropriate solvents. ${ }^{205}$

Based on that, Suh et al. ${ }^{205}$ presented a novel way in which a ligand-assisted solubility adjustment step is first applied to the colloidal solution to avoid the loss of ligands from the QD surface. In detail, a ligand solution of 4-phenylbutylamine and benzoic acid in benzene was added to $\mathrm{CsPbX}_{3}$ NC solution followed by precipitating the QDs with acetonitrile and redispersing them in benzene. Afterwards, the solid-state ligand exchange was performed on films based on these treated QDs using acid and amine ligands dissolved in octane and benzene co-solvents. Different short-chain acids and amines were tested for the exchange process. The results showed that using aromatic acid/amine ligands in the exchange process is more suitable than the acid/amine ligands based on alkyl chains. This is because a longer chain length is required in the latter case to achieve the same PL intensity as with the aromatic acid/amine ligands. Utilizing the ligand exchanged $\mathrm{CsPbX}_{3}$ QD films as photoemission layers in LEDs resulted in substantial enhancements in their efficiencies compared to their untreated counterparts due to the enhanced photoluminescence and the increased carrier mobility and coupling of lattices via the 
increased inter-particle connection when employing the short ligands. $^{205}$

Most of the other studies are based on the use of MeOAc solvent for the solid-state ligand exchange process. This goes back to the work of Swarnkar et al. ${ }^{53}$ which demonstrated a solid-state ligand exchange process for the use of $\mathrm{CsPbI}_{3}$ QD films as the photoactive layer in solar cell devices. The method is based on dipping the QD films in a saturated solution of lead nitrate $\mathrm{Pb}\left(\mathrm{NO}_{3}\right)_{2}$ or lead acetate $\mathrm{Pb}(\mathrm{OAc})_{2}$ dissolved in MeOAc. After repeating this step three to five times, the films are washed with MeOAc solution and 100 to $400 \mathrm{~nm}$ thick QD films are obtained. The fabricated QD films exhibited efficient electron transport between the dots while retaining phase stability of the QDs. Solar cells based on these were stable during operation and had a high power conversion efficiency (PCE) of $10.77 \%$ and an open-circuit voltage $\left(V_{\text {oc }}\right)$ of $1.23 \mathrm{~V}$. The films were also explored in LED devices where a bright red light was emitted with low turn-on voltage close to its bandgap. ${ }^{53}$

In 2017, the same research group demonstrated the growth of $\mathrm{CsPbI}_{3}$ QD films via the procedure used by Swarnkar et al. ${ }^{53}$ but with employing an additional step at the end of film fabrication. ${ }^{206}$ In this last step, the films were post-treated with $\mathrm{AX}$ salts in ethyl acetate (EtOAc), where A is FA, MA or Cs and X is $\mathrm{Br}$ or I (Fig. 17). The authors found that the FAI post treatment produced the best overall photovoltaic performance with a PCE value above $13 \%$. Based on FTIR spectra, the authors showed that the native oleate and oleylammonium ligands were removed by the FAI treatment reducing thereby the interparticle spacing between QDs. Furthermore, using time-resolved terahertz spectroscopy (TRTS), it was shown that compared to the untreated film, the FAI-treated QD films exhibited increased charge mobility and similar lifetime. Thus, the mobility-lifetime product, which is proportional to the charge carrier diffusion length, was larger in the FAI-treated film indicating an improved charge extraction. ${ }^{206}$

Wheeler et al. ${ }^{207}$ explored the surface chemistry of $\mathrm{CsPbI}_{3}$ QD films during this ligand exchange procedure. They showed that the oleate ligand was removed in the first step after washing with MeOAc antisolvent. The saturated solution of $\mathrm{Pb}\left(\mathrm{NO}_{3}\right)_{2}$ in MeOAc was shown not to directly interact with the QD surface but rather to impact the amount of water that interacts with the surface. The hydrolysis of MeOAc produces acetic acid and methanol as shown in Fig. 18a. Acetic acid protonates the oleate ligands bound to the NC surface and the

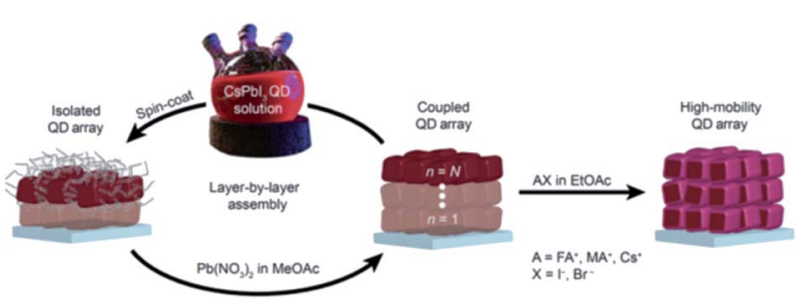

Fig. 17 Schematic representation of the deposition process of $\mathrm{CsPbl}_{3}$ QD films followed by AX treatment. The figure is taken with permission from ref. 206.

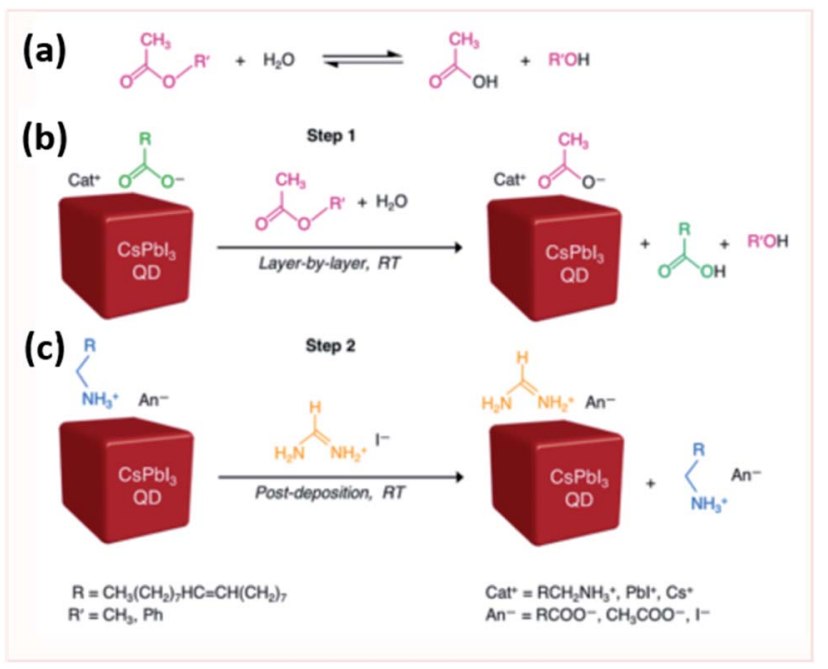

Fig. 18 Scheme showing (a) the ester hydrolysis to carboxylic acid and alcohol, (b) the anionic carboxylate ligand exchange and (c) cationic ligand exchange in $\mathrm{CsPb}_{3} \mathrm{QD}$ films. The figure is adapted with permission from ref. 207.

produced acetate binds to the surface instead (Fig. 18b). XPS, NMR and FTIR spectra proved the selective ligand exchange of oleate with acetate without affecting the composition of $\mathrm{CsPbI}_{3}$ and keeping intact the $\mathrm{OAmH}^{+}$ligands during the MeOAc washing step. These results suggested that the ligand exchange should occur under controlled relative humidity conditions (11-24\%) to obtain smooth high absorbing $\mathrm{CsPbI}_{3}$ QD films. At high humidity conditions, further ester hydrolysis occurs destabilizing the NC surface and transforming $\mathrm{CsPbI}_{3}$ NCs into the yellow phase; whereas at low humidity levels, the NC films are unevenly covered due to solvation of underlying QD layers with subsequent deposition of additional QD layers. ${ }^{207}$

After targeting the oleate ligands with the MeOAc treatment, the exposure of the QD films to FAI was shown to target the loosely bound $\mathrm{OAmH}^{+}$ligands (Fig. 18c). This resulted in an enhancement in the mobility and inter-dot electronic coupling in FAI-treated films. Indeed, FTIR, X-ray absorption spectroscopy (XAS) and time-of flight secondary ion mass spectroscopy (TOF-SIMS) indicated that the FAI treatment was selective to the surface of the NCs where $\mathrm{FA}^{+}$exchanges with $\mathrm{OAmH}^{+}$rather than the $\mathrm{Cs}^{+}$cations when the films are immersed in the FAI solution for short times. By increasing the time of treatment, the short-circuit current $\left(J_{\mathrm{sc}}\right)$ of the solar cells and, consequently, the external quantum efficiency (EQE) increase due to the loss of the insulating ligands that impede the charge transport in the films. ${ }^{207}$

Later on, this approach was extended for the deposition of layer-by-layer perovskite QD films of controlled composition to fabricate solar cells with an internal heterojunction induced by the compositional changes throughout the films. The heterojunction improved the PCE of the solar cells to $15.74 \%$ by enhancing the charge separation at the interfaces and hence improving the photocarrier collection. ${ }^{208}$ 


\subsection{Modifying the ligands in the NC synthesis}

To overcome the limitations imposed by using polar solvents during the postsynthetic and processing steps of MHP NCs, several groups have modified the NC synthesis employing new ligands that can bind more tightly to the NC surface than the typically used ones. This will result in more stable NC films which is required for good performing devices.

In one approach, the synthesis of NCs was modified to include only acid ligands. Yassitepe et al. ${ }^{209}$ reported an aminefree synthesis using tetraocylammonium halides. The obtained OA-capped NCs exhibited improved colloidal stability in polar solvents due to stronger ligand bonding compared to conventional OA/OAm capped NCs. This is because in OA/OAm-capped NCs, a rapid proton exchange occurs between the oleate and amine ligands leading to a dynamic equilibrium between the bound and unbound ligands, as discussed earlier, which limits the stability of the NCs. ${ }^{209}$ Likewise, Yan et al. ${ }^{210}$ proposed a ligand modification approach by replacing OA with 2-hexyldecanoic acid (DA) in the HI synthesis of $\mathrm{CsPbBr}_{3}$ QDs as presented in Fig. 19a. DFT calculations showed that the binding energy between this ligand with two short branched chains and the QDs is larger than of OA with the QDs. As a result, DAcapped $\mathrm{CsPbBr}_{3}$ QDs showed improved stability where no aggregation was observed after storing the QD solution in air for more than 70 days. ${ }^{210}$ Wang et al. ${ }^{211}$ reported the synthesis of $\mathrm{CsPbI}_{3} \quad \mathrm{NCs}$ using TMPPA (bis-(2,2,4-trimethylpentyl)phosphinic acid) instead of OA in the synthesis. The resulting NCs showed improved phase stability compared to the ones synthesized with OA that undergo rapid phase transition to the orthorhombic yellow phase.

Another approach is to use zwitterionic ligands to effectively retain the outstanding optoelectronic properties of colloidal NCs and achieve efficient charge carrier transport in NC film. For instance, Motti et al. ${ }^{81}$ reported the HI synthesis of $\mathrm{CsPbBr}_{3} \mathrm{NCs}$

(a)

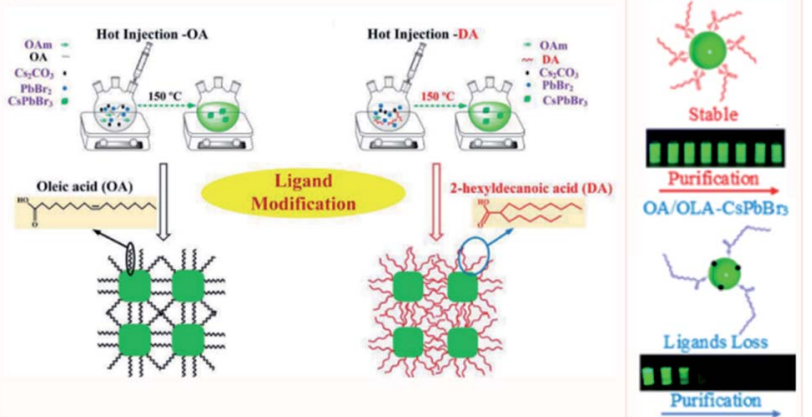

Fig. 19 (a) Schematics of the HI synthesis of $\mathrm{CsPbBr}_{3}$ QDs using two different ligands: $O A$ and DA. The $O A$ binding to the QD surface is like building blocks whereas the branched DA ligand binding to the surface is like a web interlaced together. (b) Illustration of the bonding of the surface of purified $\mathrm{CsPbBr}_{3} \mathrm{NCs}$ ligated with OPA ligand (in red) or OA/ OAm (in blue). The OPA binding stabilizes the NCs and retains the photoluminescence even after 8 purification cycles, whereas OA/OAm ligands are lost during purification resulting in a significant drop in the photoluminescence. Panel (a) and (b) are modified with permission from ref. 210 and 212, respectively. using 3-( $N, N$-dimethyloctadecylammonio)propanesulfonate and using lecithin. In addition, the authors fabricated films based on NCs capped with a dizwitterionic ligand (3,3'-(hexane-1,6diylbis(dimethylammoniumdiyl))bis propane-1-sulfonate) through solid state ligand exchange with lecithin. ${ }^{81}$ Krieg et al. ${ }^{149}$ reported stable high PLQY for films based on zwitterionic capped-CsPbBr NCs. These films exhibited high conductivities and effective charge transport leading to high current densities when employed in LED devices with a peak luminance exceeding $24000 \mathrm{~cd} \mathrm{~m}^{-2}$ and EQE of $2.5 \%$. $^{149}$

Multidentate or polymeric ligands, with both amine and carboxylic acid groups, were also used as promising alternatives of $\mathrm{OA}$ and OAm ligands since they can bind tightly as a single monolayer. ${ }^{33}$ In addition, using branched ligands such as APTES, POSS, OPA (octylphosphonic acid), TOP (trioctylphosphine) and TOPO (trioctylphosphine oxide) was shown to improve the NCs stability through the strong steric effect and strong binding to the NC surface. ${ }^{73, \mathbf{1 2 8}}$ For example, OPA-capped $\mathrm{CsPbX}_{3} \mathrm{NCs}$, showed a large enhancement in the stability compared to OA/OAm-capped NCs where the PLQY were preserved after eight purification cycles with hexane/ MeOAc (Fig. 19b). This was attributed to the strong interaction between $\mathrm{Pb}$ and OPA. ${ }^{212}$ Yang et al. ${ }^{213}$ reported the synthesis of $\mathrm{CsPBBr}_{3}$ QDs using dodecylbenzene sulfonic acid (DBSA) as a ligand. This ligand was used because of the strong bonding of the sulfonate group with the surface $\mathrm{Pb}$ ions on one hand. On another hand, it was used as a "Br-equivalent" ligand where it acts in a similar way to the $\mathrm{Br}^{-}$ions coordinating strongly to $\mathrm{Pb}$ ions in the perovskite lattice. This reduces the $\mathrm{Br}$ vacancies which act as trapping sites lowering the PLQY. As a result, the DBSA-capped QDs exhibited PLQY above 90\% even after eight purification cycles and storage for more than five months. ${ }^{213}$

A two-step surface engineering method was carried out by $\mathrm{Li}$ et $a .^{214}$ in which a short $\pi$-conjugation ligand phenethylamine (PEA) was used. In this process, OAm was partially replaced with PEA in the synthesis of $\mathrm{CsPbX}_{3}$ QDs followed by solid-state ligand exchange on the QD films using PEAX (X being $\mathrm{Br}$ or I). The resulting films showed a remarkable improvement in the charge carrier transport and a reduction in the trap states compared to untreated QD film. An EQE of 14.08\% was achieved in LED devices based on $\mathrm{CsPbI}_{3}$ NCs treated with PEA ligand. ${ }^{214}$

\subsection{Controlling ligand density on NC surface}

Besides the type of ligands used, their density on the NC surface affects the optoelectronic properties of the NC films. Insufficient ligand density lowers the PLQY and reduces the stability of the QD suspension, whereas an excess of ligands results in poor carrier injection and transport in QD films.

For an effective control of surface ligands of $\mathrm{FAPbI}_{3}$ QDs, Xue et $a .^{215}$ classified the solvents that can be used in the surface treatment into three grades based on their polarity. Grade I constitutes of highly polar solvents that can break the ionic bonds in the QDs. Grade III consists of solvents with very low polarity that they could not break the bonding of ligands to the QD surface. Therefore, grade II antisolvents with moderate 


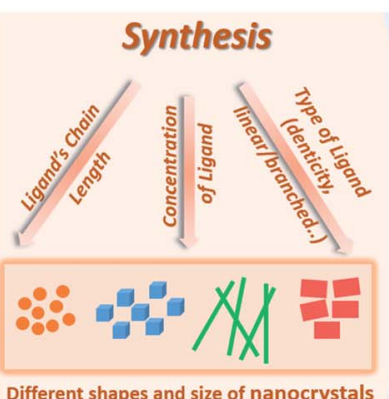

Effect of Ligands on Nanocrystals:

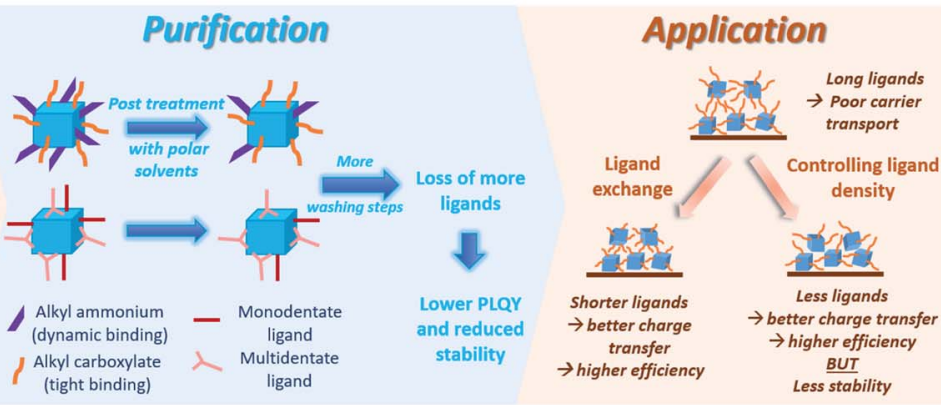

Fig. 20 A schematic showing the role of ligands during the synthesis, purification and application of perovskite NCs.

polarity are considered the best choice for effective surface treatment where the ligand density on the QD surface is dropped while preserving the structure of $\mathrm{FAPbI}_{3}$ QDs. Based on that, this group presented a surface treatment procedure consisting of two cycles in the colloidal solution followed by one treatment cycle carried out during the QD-film fabrication. In the first cycle, 2-pentanol was used as a grade II antisolvent to reduce the ligand density. Then, a lower polarity solvent was used in the second cycle to keep the structural integrity of $\mathrm{FAPbI}_{3}$ QDs and resulting in an efficient removal of ligands after two cycles. The last cycle was performed by rinsing the films with EtOAc to improve the coupling between QDs. Photovoltaic devices fabricated based on these three-cycle treated $\mathrm{FAPbI}_{3}$ QDs achieved a PCE of $8.38 \%$ with superior stability under ambient conditions. The improved EQE of the devices with the treatment cycles reflected an improved charge collection due to the decrease of ligand density and subsequent improvement in the inter-dot coupling. ${ }^{215}$

In another study, it was shown that after exploring a series of solvents the treatment of $\mathrm{CsPbBr}_{3}$ QDs using a mixed hexane/ EtOAc solvent enabled the control of ligand density on the NCs surface. ${ }^{216}$ As a result of this treatment, stable QD inks were prepared with preserved high stability enabling the growth of uniform QD films exhibiting a balance between surface passivation and carrier injection. LEDs based on these treatedQD films showed a remarkable EQE of $6.27 \%$ that corresponds to 50-fold enhancement with respect to QDs treated with one cycle of acetone. This outstanding efficiency of $\mathrm{CsPBrr}_{3}$ QD LEDs among the inorganic perovskite LEDs suggests that the performance of these devices can be improved, not only through surface ligand exchange, but also through controlling the surface ligand density. ${ }^{216}$

Annealing is another strategy that can be used to remove long chain ligands and increase the inter-particle coupling. By varying the annealing temperature, the coupling between NCs can be controlled. A post-annealing procedure of $\mathrm{CsPbBr}_{3}$ NS films was shown to partially remove the organic ligands improving thereby the coupling between NSs, the charge transfer and the produced photocurrent. ${ }^{217}$

However, to date, there is no report of a clear strategy of controlling effectively and precisely the ligand density in perovskite NCs. The optimal amount of the different types of ligands needed for both the stability and application of the NCs must be also explored.

\section{Conclusion and outlook}

Perovskite NCs exhibit promising properties such as optical tunability through size and composition modification, defect tolerance, high PLQY and ease of synthesis. Different approaches have been used to synthesize MHP NCs, most of which rely on capping ligands. The effect of ligands on the properties of NCs during their synthesis, purification and application is summarized in Fig. 20.

It was shown that both acidic (carboxylic acids) and basic (amines) ligands are essential in stabilizing the NCs and determining their morphological and optical properties. The size, shape and structure of the NCs depend on the type of used ligand, the length and bulkiness of its hydrocarbon chain, and its concentration with respect to the precursors, where the size generally decreases with higher ligand concentration or longer alkyl chain of the ligand.

Surface studies on perovskite NCs have shown that the NCligand interaction is generally weak and dynamic which leads to the desorption of the ligands upon purification of the NCs. As a consequence, the stability and photoelectric properties of the NCs are reduced after purification. Another challenge is that the commonly used ligands in the NC synthesis are long alkyl chain carboxylic acids and amines. These insulating ligands hinder the charge transport throughout the NC-based films and increase the barrier for carrier injection at the interfaces in optoelectronic devices based on these NCs.

So far, research efforts have been directed to face these challenges by considering several strategies including: (1) a careful choice of the antisolvent used in the purification of the NCs and in the NC film deposition, (2) ligand exchange to replace the insulating and weak-binding ligands with ligands that enable a stronger bonding to the NC surface and better charge transport in the NC films, and (3) ligand management by introducing new ligands in the NCs synthesis or controlling the ligands density on the NC surface to meet both demands: stable NCs and efficient charge transport in NC films.

Besides these aspects that have been covered in the review, this field is open for more exhaustive studies. Most of the 
surface investigations are carried out using inorganic $\mathrm{CsPbX}_{3}$ NCs, which are not necessarily valid for other materials, while investigations related to organic LHP NCs and lead-free MHP NCs are still missing. Hence, a logical development in this direction would be to extend these studies to other MHP NCs (organic LHP and lead-free MHP NCs) to achieve a deeper understanding of the surface termination and ligation in MHP NCs. Furthermore, there is still plenty of room to explore new ligands (such as inorganic ligands) aiming to attain a better stabilization of the NCs and a more efficient application in devices. These investigations will be possible due to the availability and advancements in the characterization techniques (e.g. NMR) and theoretical calculations.

Another research pillar is to unveil the role of ligands in the early stages of NC synthesis and have a deeper insight into the nucleation and growth of NCs. This would enable a better control of the kinetics and shape evolution during the NC synthesis. Microfluidic methods had been used to follow the early formation of LHP NCs and enable a fast screening of reaction conditions. ${ }^{\mathbf{6 0 , 1 6 7 , 2 1 8}}$ This strategy can be elaborated to investigate other MHP NCs and to monitor a broader range of reaction conditions including the use of new ligands and solvents.

One of the main research frontiers is to find alternative deposition techniques for fabricating stable NC thin films that can be directly applied in devices. MHP NC films are typically deposited by spincoating since this allows a uniform film coverage. However, to obtain films that are sufficiently thick for applications, several cycles of NC deposition and ligand exchange and washing are needed. Besides being time consuming, with every deposition cycle the used antisolvents can wash off some the previously deposited NCs, which makes it tricky to control the thickness of the films and to obtain reproducible devices afterwards. Therefore, finding methods which enable the growth of thick and uniform films based on MHP NCs remains an open issue that must be resolved in the upcoming years. More studies should also investigate how to improve the packing between NCs to enhance the charge transport in films. This can be achieved by thorough investigations of different ligands and processing steps (e.g. annealing) during the NC film fabrication. Moreover, investigating the interface between the NC film and the different layers in optoelectronic devices (such as electron transport layers and hole transport layers) will be essential to eliminate possible charge losses and to obtain devices with outperforming efficiencies and stability.

Other issues that still need to be understood and can be used to outline the future guidelines of research in this field include: optimizing the post-treatment steps for every system including the purification and the ligand exchange by using less aggressive procedures, exploring different combinations of A-, B-site cations and halides that can replace LHP NCs and outperform them in terms of stability and optoelectronic properties and investigating the effect of ligation on the excitonic properties of NCs. Also, understanding ligation in mixed perovskite NC systems would be interesting for future studies as it was shown that surface ligands play a critical role in the A-site cation exchange of LHP QDs. ${ }^{219}$

Furthermore, as discussed earlier, the issue of stability remains a main obstacle for the efficient utilization of MHP NCs. In this respect, incorporating MHP NCs in heterostructures to protect them against the harsh environmental conditions is one strategy that has been explored. However, a full understanding of the interface of the perovskite with other materials in these heterostructures and the efficiency of such structures in stabilizing the NCs on long term basis remains scarcely studied. In addition, the synthesis of a core/shell nanostructure, which allows a complete protection of the perovskite NC is still challenging and is a triggering future research area.

By understanding the surface chemistry and managing the ligand stabilization of perovskite nanocrystals, these materials may find eventual uses in solar energy and energy efficient lighting.

\section{Author contributions}

Conceptualization: all authors. Visualization and writing original draft: Fatima Haydous. Supervision, funding acquisition and project administration: James M. Gardner and Ute B. Cappel. Writing - review \& editing: all authors.

\section{Conflicts of interest}

There are no conflicts to declare.

\section{Acknowledgements}

We acknowledge research funding from the Swedish Research Council (Grant No. VR 2018-04125), the Olle Engkvist Foundation and the Göran Gustafsson Foundation. James Gardner gratefully acknowledges support from the Swedish Government through the research initiative STandUP for ENERGY.

\section{References}

1 W. R. Tilluck, A. L. Morris, J. K. Gurchiek, A. D. Evans and P. G. Van Patten, RSC Adv., 2016, 6, 81780-81788.

2 P. Moroz, L. Royo Romero and M. Zamkov, Chem. Commun., 2019, 55, 3033-3048.

3 H. Huang, A. S. Susha, S. V. Kershaw, T. F. Hung and A. L. Rogach, Adv. Sci., 2015, 2, 1500194.

4 P.-J. Lu, S.-C. Huang, Y.-P. Chen, L.-C. Chiueh and D. Y.-C. Shih, J. Food Drug Anal., 2015, 23, 587-594.

5 L. Mu and R. L. Sprando, Pharm. Res., 2010, 27, 1746-1749.

6 J. Zhou, Y. Liu, J. Tang and W. Tang, Mater. Today, 2017, 20, 360-376.

7 O. V. Salata, J. Nanobiotechnol., 2004, 2, 3.

8 S. Priyadarsini, S. Mohanty, S. Mukherjee, S. Basu and M. Mishra, J. Nanostruct. Chem., 2018, 8, 123-137.

9 H.-C. Wang, Z. Bao, H.-Y. Tsai, A.-C. Tang and R.-S. Liu, Small, 2018, 14, 1702433. 
10 Y. Zhang, R. Sun, X. Ou, K. Fu, Q. Chen, Y. Ding, L.-J. Xu, L. Liu, Y. Han, A. V. Malko, X. Liu, H. Yang, O. M. Bakr, H. Liu and O. F. Mohammed, ACS Nano, 2019, 13, 25202525.

11 S.-W. Dai, B.-W. Hsu, C.-Y. Chen, C.-A. Lee, H.-Y. Liu, H.-F. Wang, Y.-C. Huang, T.-L. Wu, A. Manikandan, R.-M. Ho, C.-S. Tsao, C.-H. Cheng, Y.-L. Chueh and H.-W. Lin, Adv. Mater., 2018, 30, 1705532.

12 S. H. Im, H.-j. Kim, S. W. Kim, S.-W. Kim and S. I. Seok, Energy Environ. Sci., 2011, 4, 4181-4186.

13 M. V. Kovalenko, L. Protesescu and M. I. Bodnarchuk, Science, 2017, 358, 745-750.

14 J. Shamsi, A. S. Urban, M. Imran, L. De Trizio and L. Manna, Chem. Rev., 2019, 119, 3296-3348.

15 M. M. Lee, J. Teuscher, T. Miyasaka, T. N. Murakami and H. J. Snaith, Science, 2012, 338, 643-647.

16 P. Fu, Q. Shan, Y. Shang, J. Song, H. Zeng, Z. Ning and J. Gong, Sci. Bull., 2017, 62, 369-380.

17 NREL, Photovoltaic Research, Best Research-Cell Efficiency Chart, accessed 19 July 2020.

18 J. Yu, G. Liu, C. Chen, Y. Li, M. Xu, T. Wang, G. Zhao and L. Zhang, J. Mater. Chem. C, 2020, 8, 6326-6341.

19 C.-Y. Chang, A. N. Solodukhin, S.-Y. Liao, K. P. O. Mahesh, C.-L. Hsu, S. A. Ponomarenko, Y. N. Luponosov and Y.-C. Chao, J. Mater. Chem. C, 2019, 7, 8634-8642.

20 C. H. A. Li, Z. Zhou, P. Vashishtha and J. E. Halpert, Chem. Mater., 2019, 31, 6003-6032.

21 J. Miao and F. Zhang, J. Mater. Chem. C, 2019, 7, 1741-1791.

22 M. M. Stylianakis, T. Maksudov, A. Panagiotopoulos, G. Kakavelakis and K. Petridis, Materials, 2019, 12, 859.

23 Z. Wei and J. Xing, J. Phys. Chem. Lett., 2019, 10, 3035-3042.

24 C. Xie, C.-K. Liu, H.-L. Loi and F. Yan, Adv. Funct. Mater., 2020, 30, 1903907.

25 T. T. Ngo and I. Mora-Seró, J. Phys. Chem. Lett., 2019, 10, 1099-1108.

26 M. Leng, Y. Yang, K. Zeng, Z. Chen, Z. Tan, S. Li, J. Li, B. Xu, D. Li, M. P. Hautzinger, Y. Fu, T. Zhai, L. Xu, G. Niu, S. Jin and J. Tang, Adv. Funct. Mater., 2018, 28, 1704446.

27 H. Huang, M. I. Bodnarchuk, S. V. Kershaw, M. V. Kovalenko and A. L. Rogach, ACS Energy Lett., 2017, 2, 2071-2083.

28 Q. A. Akkerman, G. Rainò, M. V. Kovalenko and L. Manna, Nat. Mater., 2018, 17, 394-405.

29 W. J. Mir, T. Sheikh, H. Arfin, Z. Xia and A. Nag, NPG Asia Mater., 2020, $12,9$.

30 A. Rubino, L. Caliò, A. García-Bennett, M. E. Calvo and H. Míguez, Adv. Opt. Mater., 2020, 8, 1901868.

31 Q. Fan, G. V. Biesold-McGee, J. Ma, Q. Xu, S. Pan, J. Peng and Z. Lin, Angew. Chem., Int. Ed., 2020, 59, 1030-1046.

32 M. Green, J. Organomet. Chem., 1995, 500, 127-148.

33 J. De Roo, M. Ibáñez, P. Geiregat, G. Nedelcu, W. Walravens, J. Maes, J. C. Martins, I. Van Driessche, M. V. Kovalenko and Z. Hens, ACS Nano, 2016, 10, 2071-2081.

34 N. Kirkwood, J. O. V. Monchen, R. W. Crisp, G. Grimaldi, H. A. C. Bergstein, I. du Fossé, W. van der Stam, I. Infante and A. J. Houtepen, J. Am. Chem. Soc., 2018, 140, 1571215723.
35 K. Xu, C. C. Lin, X. Xie and A. Meijerink, Chem. Mater., 2017, 29, 4265-4272.

36 J. Pan, Y. Shang, J. Yin, M. De Bastiani, W. Peng, I. Dursun, L. Sinatra, A. M. El-Zohry, M. N. Hedhili, A.-H. Emwas, O. F. Mohammed, Z. Ning and O. M. Bakr, J. Am. Chem. Soc., 2018, 140, 562-565.

37 H. Takeuchi, B. Omogo and C. D. Heyes, Nano Lett., 2013, 13, 4746-4752.

38 B. Lv, Y. Xu, D. Wu and Y. Sun, Chem. Commun., 2011, 47, 967-969.

39 A. Klein, A. Kaiser, W. Wielandt, F. Belaj, E. Wendel, H. Bertagnolli and S. Záliš, Inorg. Chem., 2008, 47, 1132411333.

40 A. Dehno Khalaji, M. Weil, H. Hadadzadeh and M. Daryanavard, Inorg. Chim. Acta, 2009, 362, 4837-4842.

41 B. N. Sivasankar and S. Govindarajan, Synthesis and Reactivity in Inorganic and Metal-Organic Chemistry, 1995, 25, pp. 31-43.

42 S. Govindarajan, K. C. Patil, H. Manohar and P.-E. Werner, J. Chem. Soc., Dalton Trans., 1986, 119-123, DOI: 10.1039/ DT9860000119.

43 E. Waldhör, W. Kaim, J. A. Olabe, L. D. Slep and J. Fiedler, Inorg. Chem., 1997, 36, 2969-2974.

44 A. Nag, M. V. Kovalenko, J.-S. Lee, W. Liu, B. Spokoyny and D. V. Talapin, J. Am. Chem. Soc., 2011, 133, 10612-10620.

45 M. Mittal, A. Jana, S. Sarkar, P. Mahadevan and S. Sapra, J. Phys. Chem. Lett., 2016, 7, 3270-3277.

46 D. Chen, X. Chen, Z. Wan and G. Fang, ACS Appl. Mater. Interfaces, 2017, 9, 20671-20678.

47 B. Xu, W. Wang, X. Zhang, W. Cao, D. Wu, S. Liu, H. Dai, S. Chen, K. Wang and X. Sun, J. Mater. Chem. C, 2018, 6, 668.

48 L. Protesescu, S. Yakunin, S. Kumar, J. Bär, F. Bertolotti, N. Masciocchi, A. Guagliardi, M. Grotevent, I. Shorubalko, M. I. Bodnarchuk, C.-J. Shih and M. V. Kovalenko, ACS Nano, 2017, 11, 3119-3134.

49 Y.-F. Li, J. Feng and H.-B. Sun, Nanoscale, 2019, 11, 1911919139.

50 J. Deng, J. Li, Z. Yang and M. Wang, J. Mater. Chem. C, 2019, 7, 12415-12440.

51 W. Travis, E. N. K. Glover, H. Bronstein, D. O. Scanlon and R. G. Palgrave, Chem. Sci., 2016, 7, 4548-4556.

52 Y. Dong, Y. Zhao, S. Zhang, Y. Dai, L. Liu, Y. Li and Q. J. Chen, J. Mater. Chem. A, 2018, 6, 21729-21746.

53 A. Swarnkar, A. R. Marshall, E. M. Sanehira, B. D. Chernomordik, D. T. Moore, J. A. Christians, T. Chakrabarti and J. M. Luther, Science, 2016, 354, 92-95.

54 C. C. Stoumpos, C. D. Malliakas and M. G. Kanatzidis, Inorg. Chem., 2013, 52, 9019-9038.

55 K. Hong, Q. V. Le, S. Y. Kim and H. W. Jang, J. Mater. Chem. $C$, 2018, 6, 2189-2209.

56 M. I. Saidaminov, O. F. Mohammed and O. M. Bakr, ACS Energy Lett., 2017, 2, 889-896.

57 L. Protesescu, S. Yakunin, M. I. Bodnarchuk, F. Krieg, R. Caputo, C. H. Hendon, R. X. Yang, A. Walsh and M. V. Kovalenko, Nano Lett., 2015, 15, 3692-3696. 
58 F. Zhang, H. Zhong, C. Chen, X.-g. Wu, X. Hu, H. Huang, J. Han, B. Zou and Y. Dong, ACS Nano, 2015, 9, 4533-4542.

59 L. Protesescu, S. Yakunin, M. I. Bodnarchuk, F. Bertolotti, N. Masciocchi, A. Guagliardi and M. V. Kovalenko, J. Am. Chem. Soc., 2016, 138, 14202-14205.

60 I. Lignos, L. Protesescu, D. B. Emiroglu, R. Maceiczyk, S. Schneider, M. V. Kovalenko and A. J. deMello, Nano Lett., 2018, 18, 1246-1252.

61 D. N. Minh, J. Kim, J. Hyon, J. H. Sim, H. H. Sowlih, C. Seo, J. Nam, S. Eom, S. Suk, S. Lee, E. Kim and Y. Kang, Chem. Mater., 2017, 29, 5713-5719.

62 T. Umebayashi, K. Asai, T. Kondo and A. Nakao, Phys. Rev. $B, 2003,67,155405$.

63 V. K. Ravi, G. B. Markad and A. Nag, ACS Energy Lett., 2016, 1, 665-671.

64 T. C. Jellicoe, J. M. Richter, H. F. J. Glass, M. Tabachnyk, R. Brady, S. E. Dutton, A. Rao, R. H. Friend, D. Credgington, N. C. Greenham and M. L. Böhm, J. Am. Chem. Soc., 2016, 138, 2941-2944.

65 P. Arunkumar, K. H. Gil, S. Won, S. Unithrattil, Y. H. Kim, H. J. Kim and W. B. Im, J. Phys. Chem. Lett., 2017, 8, 4161-4166.

66 G. E. Eperon, S. D. Stranks, C. Menelaou, M. B. Johnston, L. M. Herz and H. J. Snaith, Energy Environ. Sci., 2014, 7, 982-988.

67 S. Meloni, G. Palermo, N. Ashari-Astani, M. Grätzel and U. Rothlisberger, J. Mater. Chem. A, 2016, 4, 15997-16002.

68 Y. Tong, E. Bladt, M. F. Aygüler, A. Manzi, K. Z. Milowska, V. A. Hintermayr, P. Docampo, S. Bals, A. S. Urban, L. Polavarapu and J. Feldmann, Angew. Chem., Int. Ed., 2016, 55, 13887-13892.

69 S. Gonzalez-Carrero, L. Francés-Soriano, M. González-Béjar, S. Agouram, R. E. Galian and J. Pérez-Prieto, Small, 2016, 12, 5245-5250.

70 M. Imran, V. Caligiuri, M. Wang, L. Goldoni, M. Prato, R. Krahne, L. De Trizio and L. Manna, J. Am. Chem. Soc., 2018, 140, 2656-2664.

71 I. Levchuk, A. Osvet, X. Tang, M. Brandl, J. D. Perea, F. Hoegl, G. J. Matt, R. Hock, M. Batentschuk and C. J. Brabec, Nano Lett., 2017, 17, 2765-2770.

72 B. A. Koscher, J. K. Swabeck, N. D. Bronstein and A. P. Alivisatos, J. Am. Chem. Soc., 2017, 139, 6566-6569.

73 F. Liu, Y. Zhang, C. Ding, S. Kobayashi, T. Izuishi, N. Nakazawa, T. Toyoda, T. Ohta, S. Hayase, T. Minemoto, K. Yoshino, S. Dai and Q. Shen, ACS Nano, 2017, 11, 10373-10383.

74 Y. Peter and M. Cardona, Fundamentals of Semiconductors: Physics and Materials Properties, Springer Science \& Business Media, 2010.

75 K. Tanaka, T. Takahashi, T. Ban, T. Kondo, K. Uchida and N. Miura, Solid State Commun., 2003, 127, 619-623.

76 J. Shamsi, A. S. Urban, M. Imran, L. De Trizio and L. Manna, Chem. Rev., 2019, 119, 3296-3348.

77 J. Even, L. Pedesseau and C. Katan, J. Phys. Chem. C, 2014, 118, 11566-11572.

78 M. Saba, M. Cadelano, D. Marongiu, F. Chen, V. Sarritzu, N. Sestu, C. Figus, M. Aresti, R. Piras, A. Geddo Lehmann,
C. Cannas, A. Musinu, F. Quochi, A. Mura and G. Bongiovanni, Nat. Commun., 2014, 5, 5049.

79 E. Menéndez-Proupin, P. Palacios, P. Wahnón and J. C. Conesa, Phys. Rev. B: Condens. Matter Mater. Phys., 2014, 90, 045207.

80 J. Li, L. Luo, H. Huang, C. Ma, Z. Ye, J. Zeng and H. He, J. Phys. Chem. Lett., 2017, 8, 1161-1168.

81 S. G. Motti, F. Krieg, A. J. Ramadan, J. B. Patel, H. J. Snaith, M. V. Kovalenko, M. B. Johnston and L. M. Herz, Adv. Funct. Mater., 2020, 30, 1909904.

82 Y. Shynkarenko, M. I. Bodnarchuk, C. Bernasconi, Y. Berezovska, V. Verteletskyi, S. T. Ochsenbein and M. V. Kovalenko, ACS Energy Lett., 2019, 4, 2703-2711.

83 W.-J. Yin, T. Shi and Y. Yan, Appl. Phys. Lett., 2014, 104, 063903.

84 J. M. Ball and A. Petrozza, Nat. Energy, 2016, 1, 16149.

85 S. Ten Brinck, F. Zaccaria and I. Infante, ACS Energy Lett., 2019, 4, 2739-2747.

86 J. Kang and L.-W. Wang, J. Phys. Chem. Lett., 2017, 8, 489493.

87 Y. Guo, Q. Wang and W. A. Saidi, J. Phys. Chem. C, 2017, 121, 1715-1722.

88 L. C. Schmidt, A. Pertegás, S. González-Carrero, O. Malinkiewicz, S. Agouram, G. Mínguez Espallargas, H. J. Bolink, R. E. Galian and J. Pérez-Prieto, J. Am. Chem. Soc., 2014, 136, 850-853.

89 L. Yuan, R. Patterson, X. Wen, Z. Zhang, G. Conibeer and S. Huang, J. Colloid Interface Sci., 2017, 504, 586-592.

90 T. Leijtens, G. E. Eperon, N. K. Noel, S. N. Habisreutinger, A. Petrozza and H. J. Snaith, Adv. Energy Mater., 2015, 5, 1500963.

91 T. A. Berhe, W.-N. Su, C.-H. Chen, C.-J. Pan, J.-H. Cheng, H.-M. Chen, M.-C. Tsai, L.-Y. Chen, A. A. Dubale and B.-J. Hwang, Energy Environ. Sci., 2016, 9, 323-356.

92 N. Aristidou, C. Eames, I. Sanchez-Molina, X. Bu, J. Kosco, M. S. Islam and S. A. Haque, Nat. Commun., 2017, 8, 15218.

93 S. Hou, Y. Guo, Y. Tang and Q. Quan, ACS Appl. Mater. Interfaces, 2017, 9, 18417-18422.

94 M. Meyns, M. Perálvarez, A. Heuer-Jungemann, W. Hertog, M. Ibáñez, R. Nafria, A. Genç, J. Arbiol, M. V. Kovalenko, J. Carreras, A. Cabot and A. G. Kanaras, ACS Appl. Mater. Interfaces, 2016, 8, 19579-19586.

95 S. N. Raja, Y. Bekenstein, M. A. Koc, S. Fischer, D. Zhang, L. Lin, R. O. Ritchie, P. Yang and A. P. Alivisatos, ACS Appl. Mater. Interfaces, 2016, 8, 35523-35533.

96 A. N. Aleshin, I. P. Shcherbakov, E. V. Gushchina, L. B. Matyushkin and V. A. Moshnikov, Org. Electron., 2017, 50, 213-219.

97 J. He, H. Chen, H. Chen, Y. Wang, S.-T. Wu and Y. Dong, Opt. Express, 2017, 25, 12915-12925.

98 K. Ma, X.-Y. Du, Y.-W. Zhang and S. Chen, J. Mater. Chem. C, 2017, 5, 9398-9404.

99 L. Xi, C. B. Boothroyd, T. Salim, S. Borghardt, Y. M. Lam and B. E. Kardynał, J. Mater. Chem. C, 2017, 5, 7207-7214.

100 H. An, W. K. Kim, C. Wu and T. W. Kim, Org. Electron., 2018, 56, 41-45. 
101 X. Li, Z. Xue, D. Luo, C. Huang, L. Liu, X. Qiao, C. Liu, Q. Song, C. Yan, Y. Li and T. Wang, Sci. China Mater., 2018, 61, 363-370.

102 Y. Xin, H. Zhao and J. Zhang, ACS Appl. Mater. Interfaces, 2018, 10, 4971-4980.

103 S. Yang, F. Zhang, J. Tai, Y. Li, Y. Yang, H. Wang, J. Zhang, Z. Xie, B. Xu, H. Zhong, K. Liu and B. Yang, Nanoscale, 2018, 10, 5820-5826.

104 M. Zhang, M. Wang, Z. Yang, J. Li and H. Qiu, J. Alloys Compd., 2018, 748, 537-545.

105 H. Zhang, X. Wang, Q. Liao, Z. Xu, H. Li, L. Zheng and H. Fu, Adv. Funct. Mater., 2017, 27, 1604382.

106 Q. Wang, Z. Jin, D. Chen, D. Bai, H. Bian, J. Sun, G. Zhu, G. Wang and S. Liu, Adv. Energy Mater., 2018, 8, 1800007.

107 Z. Tan, Y. Wu, H. Hong, J. Yin, J. Zhang, L. Lin, M. Wang, X. Sun, L. Sun, Y. Huang, K. Liu, Z. Liu and H. Peng, J. Am. Chem. Soc., 2016, 138, 16612-16615.

108 D.-H. Kwak, D.-H. Lim, H.-S. Ra, P. Ramasamy and J.-S. Lee, RSC Adv., 2016, 6, 65252-65256.

109 A. Pan, M. J. Jurow, F. Qiu, J. Yang, B. Ren, J. J. Urban, L. He and Y. Liu, Nano Lett., 2017, 17, 6759-6765.

110 Y.-F. Xu, M.-Z. Yang, B.-X. Chen, X.-D. Wang, H.-Y. Chen, D.-B. Kuang and C.-Y. Su, J. Am. Chem. Soc., 2017, 139, 5660-5663.

111 Q. Wang, L. Tao, X. Jiang, M. Wang and Y. Shen, Appl. Surf. Sci., 2019, 465, 607-613.

112 J.-S. Chen, T. L. Doane, M. Li, H. Zang, M. M. Maye and M. Cotlet, Part. Part. Syst. Charact., 2018, 35, 1700310.

113 H. Huang, J. Li, Y. Yi, J. Wang, Y. Kang, P. K. Chu, H. C. Ong and X.-F. Yu, Chem. Commun., 2018, 54, 2365-2368.

114 H. Li, X. Zheng, Y. Liu, Z. Zhang and T. Jiang, Nanoscale, 2018, 10, 1650-1659.

115 S. Muduli, P. Pandey, G. Devatha, R. Babar, T. M, D. C. Kothari, M. Kabir, P. P. Pillai and S. Ogale, Angew. Chem., 2018, 57, 7682-7686.

116 Y. Chen, M. Yu, S. Ye, J. Song and J. Qu, Nanoscale, 2018, 10, 6704-6711.

117 X. Di, J. Jiang, Z. Hu, L. Zhou, P. Li, S. Liu, W. Xiang and X. Liang, Dyes Pigm., 2017, 146, 361-367.

118 L. Xu, J. Chen, J. Song, J. Li, J. Xue, Y. Dong, B. Cai, Q. Shan, B. Han and H. Zeng, ACS Appl. Mater. Interfaces, 2017, 9, 26556-26564.

119 Z. Hu, Z. Liu, Y. Bian, S. Li, X. Tang, J. Du, Z. Zang, M. Zhou, W. Hu, Y. Tian and Y. Leng, Adv. Opt. Mater., 2018, 6, 1700997.

120 H. C. Yoon, S. Lee, J. K. Song, H. Yang and Y. R. Do, ACS Appl. Mater. Interfaces, 2018, 10, 11756-11767.

121 S. Demchyshyn, J. M. Roemer, H. Groiß, H. Heilbrunner, C. Ulbricht, D. Apaydin, A. Böhm, U. Rütt, F. Bertram, G. Hesser, M. C. Scharber, N. S. Sariciftci, B. Nickel, S. Bauer, E. D. Głowacki and M. Kaltenbrunner, Sci. Adv., 2017, 3, e1700738.

122 Z. Li, L. Kong, S. Huang and L. Li, Angew. Chem., 2017, 56, 8134-8138.

123 M. He, Y. Cheng, R. Yuan, L. Zhou, J. Jiang, T. Xu, W. Chen, Z. Liu, W. Xiang and X. Liang, Dyes Pigm., 2018, 152, 146154.
124 A. Loiudice, S. Saris, E. Oveisi, D. T. L. Alexander and R. Buonsanti, Angew. Chem., 2017, 56, 10696-10701.

125 C. Sun, X. Shen, Y. Zhang, Y. Wang, X. Chen, C. Ji, H. Shen, H. Shi, Y. Wang and W. W. Yu, Nanotechnology, 2017, 28, 365601.

126 M. He, Y. Cheng, L. Shen, C. Shen, H. Zhang, W. Xiang and X. Liang, Appl. Surf. Sci., 2018, 448, 400-406.

127 Q. Shan, J. Song, Y. Zou, J. Li, L. Xu, J. Xue, Y. Dong, B. Han, J. Chen and H. Zeng, Small, 2017, 13, 1701770.

128 D. Yang, X. Li and H. Zeng, Adv. Mater. Interfaces, 2018, 5, 1701662.

129 C. Murray, D. J. Norris and M. G. Bawendi, J. Am. Chem. Soc., 1993, 115, 8706-8715.

130 W. W. Yu and X. Peng, Angew. Chem., Int. Ed., 2002, 41, 2368-2371.

131 C. R. Bullen and P. Mulvaney, Nano Lett., 2004, 4, 23032307.

132 J. Zhang, J. Gao, E. M. Miller, J. M. Luther and M. C. Beard, ACS Nano, 2013, 8, 614-622.

133 O. Vybornyi, S. Yakunin and M. V. Kovalenko, Nanoscale, 2016, 8, 6278-6283.

134 J. Pal, S. Manna, A. Mondal, S. Das, K. V. Adarsh and A. Nag, Angew. Chem., Int. Ed., 2017, 56, 14187-14191.

135 A. Kostopoulou, E. Kymakis and E. Stratakis, J. Mater. Chem. A, 2018, 6, 9765-9798.

136 Y. Bekenstein, B. A. Koscher, S. W. Eaton, P. Yang and A. P. Alivisatos, J. Am. Chem. Soc., 2015, 137, 16008-16011.

137 D. Yang, Y. Zou, P. Li, Q. Liu, L. Wu, H. Hu, Y. Xu, B. Sun, Q. Zhang and S.-T. Lee, Nano Energy, 2018, 47, 235-242.

138 D. Zhang, S. W. Eaton, Y. Yu, L. Dou and P. Yang, J. Am. Chem. Soc., 2015, 137, 9230-9233.

139 Y. Dong, T. Qiao, D. Kim, D. Parobek, D. Rossi and D. H. Son, Nano Lett., 2018, 18, 3716-3722.

140 A. Dutta, S. K. Dutta, S. Das Adhikari and N. Pradhan, ACS Energy Lett., 2018, 3, 329-334.

141 A. Pan, B. He, X. Fan, Z. Liu, J. J. Urban, A. P. Alivisatos, L. He and Y. Liu, ACS Nano, 2016, 10, 7943-7954.

142 J. Song, L. Xu, J. Li, J. Xue, Y. Dong, X. Li and H. Zeng, Adv. Mater., 2016, 28, 4861-4869.

143 G. Almeida, L. Goldoni, Q. Akkerman, Z. Dang, A. H. Khan, S. Marras, I. Moreels and L. Manna, ACS Nano, 2018, 12, 1704-1711.

144 H. Huang, J. Raith, S. V. Kershaw, S. Kalytchuk, O. Tomanec, L. Jing, A. S. Susha, R. Zboril and A. L. Rogach, Nat. Commun., 2017, 8, 996.

145 M. Imran, F. Di Stasio, Z. Dang, C. Canale, A. H. Khan, J. Shamsi, R. Brescia, M. Prato and L. Manna, Chem. Mater., 2016, 28, 6450-6454.

146 J. Shamsi, Z. Dang, P. Bianchini, C. Canale, F. Di Stasio, R. Brescia, M. Prato and L. Manna, J. Am. Chem. Soc., 2016, 138, 7240-7243.

147 J. H. Park, A.-y. Lee, J. C. Yu, Y. S. Nam, Y. Choi, J. Park and M. H. Song, ACS Appl. Mater. Interfaces, 2019, 11, 84288435 .

148 S. A. Veldhuis, Y. K. E. Tay, A. Bruno, S. S. H. Dintakurti, S. Bhaumik, S. K. Muduli, M. Li, N. Mathews, T. C. Sum and S. G. Mhaisalkar, Nano Lett., 2017, 17, 7424-7432. 
149 F. Krieg, S. T. Ochsenbein, S. Yakunin, S. Ten Brinck, P. Aellen, A. Süess, B. Clerc, D. Guggisberg, O. Nazarenko, Y. Shynkarenko, S. Kumar, C.-J. Shih, I. Infante and M. V. Kovalenko, ACS Energy Lett., 2018, 3, 641-646.

150 H. Yang, Y. Zhang, K. Hills-Kimball, Y. Zhou and O. Chen, Sustainable Energy Fuels, 2018, 2, 2381-2397.

151 X. Li, F. Cao, D. Yu, J. Chen, Z. Sun, Y. Shen, Y. Zhu, L. Wang, Y. Wei, Y. Wu and H. Zeng, Small, 2017, 13, 1603996.

152 M. A. Malik, M. Y. Wani and M. A. Hashim, Arabian J. Chem., 2012, 5, 397-417.

153 S. Sun, D. Yuan, Y. Xu, A. Wang and Z. Deng, ACS Nano, 2016, 10, 3648-3657.

154 F. Zhang, S. Huang, P. Wang, X. Chen, S. Zhao, Y. Dong and H. Zhong, Chem. Mater., 2017, 29, 3793-3799.

155 I. Levchuk, P. Herre, M. Brandl, A. Osvet, R. Hock, W. Peukert, P. Schweizer, E. Spiecker, M. Batentschuk and C. J. Brabec, Chem. Commun., 2017, 53, 244-247.

156 M. B. Teunis, M. A. Johnson, B. B. Muhoberac, S. Seifert and R. Sardar, Chem. Mater., 2017, 29, 3526-3537.

157 J. Cho, Y.-H. Choi, T. E. O'Loughlin, L. De Jesus and S. Banerjee, Chem. Mater., 2016, 28, 6909-6916.

158 J. A. Sichert, Y. Tong, N. Mutz, M. Vollmer, S. Fischer, K. Z. Milowska, R. García Cortadella, B. Nickel, C. Cardenas-Daw, J. K. Stolarczyk, A. S. Urban and J. Feldmann, Nano Lett., 2015, 15, 6521-6527.

159 X. Chen, D. Chen, J. Li, G. Fang, H. Sheng and J. Zhong, Dalton Trans., 2018, 47, 5670-5678.

160 B. Luo, S. B. Naghadeh, A. L. Allen, X. Li and J. Z. Zhang, Adv. Funct. Mater., 2017, 27, 1604018.

161 B. Luo, Y.-C. Pu, S. A. Lindley, Y. Yang, L. Lu, Y. Li, X. Li and J. Z. Zhang, Angew. Chem., Int. Ed., 2016, 55, 8864-8868.

162 H. Huang, F. Zhao, L. Liu, F. Zhang, X.-g. Wu, L. Shi, B. Zou, Q. Pei and H. Zhong, ACS Appl. Mater. Interfaces, 2015, 7, 28128-28133.

163 Y.-H. Kim, G.-H. Lee, Y.-T. Kim, C. Wolf, H. J. Yun, W. Kwon, C. G. Park and T. W. Lee, Nano Energy, 2017, 38, 51-58.

164 Q. A. Akkerman, S. G. Motti, A. R. Srimath Kandada, E. Mosconi, V. D'Innocenzo, G. Bertoni, S. Marras, B. A. Kamino, L. Miranda, F. De Angelis, A. Petrozza, M. Prato and L. Manna, J. Am. Chem. Soc., 2016, 138, 1010-1016.

165 X.-D. Liu, Q. Wang, Z.-Q. Cheng, Y.-H. Qiu, L. Zhou and Q.-Q. Wang, Mater. Lett., 2017, 206, 75-79.

166 F. Fang, W. Chen, Y. Li, H. Liu, M. Mei, R. Zhang, J. Hao, M. Mikita, W. Cao, R. Pan, K. Wang and X. W. Sun, Adv. Funct. Mater., 2018, 28, 1706000.

167 I. Lignos, S. Stavrakis, G. Nedelcu, L. Protesescu, A. J. deMello and M. V. Kovalenko, Nano Lett., 2016, 16, 1869-1877.

168 Q. Pan, H. Hu, Y. Zou, M. Chen, L. Wu, D. Yang, X. Yuan, J. Fan, B. Sun and Q. Zhang, J. Mater. Chem. C, 2017, 5, 10947-10954.

169 J. Shamsi, P. Rastogi, V. Caligiuri, A. L. Abdelhady, D. Spirito, L. Manna and R. Krahne, ACS Nano, 2017, 11, 10206-10213.
170 Z. Long, H. Ren, J. Sun, J. Ouyang and N. Na, Chem. Commun., 2017, 53, 9914-9917.

171 D. M. Jang, D. H. Kim, K. Park, J. Park, J. W. Lee and J. K. Song, J. Mater. Chem. C, 2016, 4, 10625-10629.

172 M. Chen, Y. Zou, L. Wu, Q. Pan, D. Yang, H. Hu, Y. Tan, Q. Zhong, Y. Xu, H. Liu, B. Sun and Q. Zhang, Adv. Funct. Mater., 2017, 27, 1701121.

173 H. Liu, Z. Wu, H. Gao, J. Shao, H. Zou, D. Yao, Y. Liu, H. Zhang and B. Yang, ACS Appl. Mater. Interfaces, 2017, 9, 42919-42927.

174 Y. Tong, B. J. Bohn, E. Bladt, K. Wang, P. MüllerBuschbaum, S. Bals, A. S. Urban, L. Polavarapu and J. Feldmann, Angew. Chem., Int. Ed., 2017, 56, 13887-13892.

175 J. Shamsi, Z. Dang, P. Ijaz, A. L. Abdelhady, G. Bertoni, I. Moreels and L. Manna, Chem. Mater., 2018, 30, 79-83.

176 Y. Hassan, Y. Song, R. D. Pensack, A. I. Abdelrahman, Y. Kobayashi, M. A. Winnik and G. D. Scholes, Adv. Mater., 2016, 28, 566-573.

177 Z. Chen, Z.-G. Gu, W.-Q. Fu, F. Wang and J. Zhang, ACS Appl. Mater. Interfaces, 2016, 8, 28737-28742.

178 V. Malgras, S. Tominaka, J. W. Ryan, J. Henzie, T. Takei, K. Ohara and Y. Yamauchi, J. Am. Chem. Soc., 2016, 138, 13874-13881.

179 M. Anaya, A. Rubino, T. C. Rojas, J. F. Galisteo-López, M. E. Calvo and H. Míguez, Adv. Opt. Mater., 2017, 5, 1601087.

180 H.-C. Wang, S.-Y. Lin, A.-C. Tang, B. P. Singh, H.-C. Tong, C.-Y. Chen, Y.-C. Lee, T.-L. Tsai and R.-S. Liu, Angew. Chem., Int. Ed., 2016, 55, 7924-7929.

181 M. V. Kovalenko, L. Manna, A. Cabot, Z. Hens, D. V. Talapin, C. R. Kagan, V. I. Klimov, A. L. Rogach, P. Reiss and D. J. Milliron, ACS Nano, 2015, 9, 1012-1057.

182 R. Grisorio, M. E. Di Clemente, E. Fanizza, I. Allegretta, D. Altamura, M. Striccoli, R. Terzano, C. Giannini, M. Irimia-Vladu and G. P. Suranna, Nanoscale, 2019, 11, 986-999.

183 M. I. Bodnarchuk, S. C. Boehme, S. Ten Brinck, C. Bernasconi, Y. Shynkarenko, F. Krieg, R. Widmer, B. Aeschlimann, D. Günther, M. V. Kovalenko and I. Infante, ACS Energy Lett., 2019, 4, 63-74.

184 J. Shamsi, A. L. Abdelhady, S. Accornero, M. Arciniegas, L. Goldoni, A. R. S. Kandada, A. Petrozza and L. Manna, ACS Energy Lett., 2016, 1, 1042-1048.

185 S. Ten Brinck and I. Infante, ACS Energy Lett., 2016, 1, 12661272.

186 C. Giansante and I. Infante, J. Phys. Chem. Lett., 2017, 8, 5209-5215.

187 Z.-L. Yu, Q.-R. Ma, Y.-Q. Zhao, B. Liu and M.-Q. Cai, J. Phys. Chem. C, 2018, 122, 9275-9282.

188 M. Leng, Z. Chen, Y. Yang, Z. Li, K. Zeng, K. Li, G. Niu, Y. He, Q. Zhou and J. Tang, Angew. Chem., Int. Ed., 2016, 55, 15012-15016.

189 S. Wei, Y. Yang, X. Kang, L. Wang, L. Huang and D. Pan, Chem. Commun., 2016, 52, 7265-7268.

190 J. Maes, L. Balcaen, E. Drijvers, Q. Zhao, J. De Roo, A. Vantomme, F. Vanhaecke, P. Geiregat and Z. Hens, J. Phys. Chem. Lett., 2018, 9, 3093-3097. 
191 V. K. Ravi, P. K. Santra, N. Joshi, J. Chugh, S. K. Singh, H. Rensmo, P. Ghosh and A. Nag, J. Phys. Chem. Lett., 2017, 8, 4988-4994.

192 F. Di Stasio, S. Christodoulou, N. Huo and G. Konstantatos, Chem. Mater., 2017, 29, 7663-7667.

193 F. Hu, H. Zhang, C. Sun, C. Yin, B. Lv, C. Zhang, W. W. Yu, X. Wang, Y. Zhang and M. Xiao, ACS Nano, 2015, 9, 1241012416.

194 Y. Kim, E. Yassitepe, O. Voznyy, R. Comin, G. Walters, X. Gong, P. Kanjanaboos, A. F. Nogueira and E. H. Sargent, ACS Appl. Mater. Interfaces, 2015, 7, 2500725013.

195 Q. A. Akkerman, M. Gandini, F. Di Stasio, P. Rastogi, F. Palazon, G. Bertoni, J. M. Ball, M. Prato, A. Petrozza and L. Manna, Nat. Energy, 2016, 2, 16194.

196 M. Que, L. Zhu, Y. Guo, W. Que and S. Yun, J. Mater. Chem. C, 2020, 8, 5321-5334.

197 H. Dong, C. Zhang, X. Liu, J. Yao and Y. S. Zhao, Chem. Soc. Rev., 2020, 49, 951-982.

198 X. Gong, O. Voznyy, A. Jain, W. Liu, R. Sabatini, Z. Piontkowski, G. Walters, G. Bappi, S. Nokhrin, O. Bushuyev, M. Yuan, R. Comin, D. McCamant, S. O. Kelley and E. H. Sargent, Nat. Mater., 2018, 17, 550556.

199 J. Pan, L. N. Quan, Y. Zhao, W. Peng, B. Murali, S. P. Sarmah, M. Yuan, L. Sinatra, N. M. Alyami, J. Liu, E. Yassitepe, Z. Yang, O. Voznyy, R. Comin, M. N. Hedhili, O. F. Mohammed, Z. H. Lu, D. H. Kim, E. H. Sargent and O. M. Bakr, Adv. Mater., 2016, 28, 87188725.

200 J. Pan, S. P. Sarmah, B. Murali, I. Dursun, W. Peng, M. R. Parida, J. Liu, L. Sinatra, N. Alyami, C. Zhao, E. Alarousu, T. K. Ng, B. S. Ooi, O. M. Bakr and O. F. Mohammed, J. Phys. Chem. Lett., 2015, 6, 5027-5033.

201 F. Ye, H. Zhang, W. Li, Y. Yan, J. Cai, R. S. Gurney, A. J. Pearson, D. Liu and T. Wang, Small Methods, 2019, 3, 1800489.

202 F. Palazon, G. Almeida, Q. A. Akkerman, L. De Trizio, Z. Dang, M. Prato and L. Manna, Chem. Mater., 2017, 29, 4167-4171.

203 Z. Liu, Y. Bekenstein, X. Ye, S. C. Nguyen, J. Swabeck, D. Zhang, S.-T. Lee, P. Yang, W. Ma and A. P. Alivisatos, J. Am. Chem. Soc., 2017, 139, 5309-5312.

204 S. K. Balakrishnan and P. V. Kamat, Chem. Mater., 2018, 30, 74-78.
205 Y.-H. Suh, T. Kim, J. W. Choi, C.-L. Lee and J. Park, ACS Appl. Nano Mater., 2018, 1, 488-496.

206 E. M. Sanehira, A. R. Marshall, J. A. Christians, S. P. Harvey, P. N. Ciesielski, L. M. Wheeler, P. Schulz, L. Y. Lin, M. C. Beard and J. M. Luther, Sci. Adv., 2017, 3, eaao4204.

207 L. M. Wheeler, E. M. Sanehira, A. R. Marshall, P. Schulz, M. Suri, N. C. Anderson, J. A. Christians, D. Nordlund, D. Sokaras, T. Kroll, S. P. Harvey, J. J. Berry, L. Y. Lin and J. M. Luther, J. Am. Chem. Soc., 2018, 140, 10504-10513.

208 Q. Zhao, A. Hazarika, X. Chen, S. P. Harvey, B. W. Larson, G. R. Teeter, J. Liu, T. Song, C. Xiao, L. Shaw, M. Zhang, G. Li, M. C. Beard and J. M. Luther, Nat. Commun., 2019, 10, 2842.

209 E. Yassitepe, Z. Yang, O. Voznyy, Y. Kim, G. Walters, J. A. Castañeda, P. Kanjanaboos, M. Yuan, X. Gong, F. Fan, J. Pan, S. Hoogland, R. Comin, O. M. Bakr, L. A. Padilha, A. F. Nogueira and E. H. Sargent, Adv. Funct. Mater., 2016, 26, 8757-8763.

210 D. Yan, T. Shi, Z. Zang, T. Zhou, Z. Liu, Z. Zhang, J. Du, Y. Leng and X. Tang, Small, 2019, 15, 1901173.

211 C. Wang, A. S. R. Chesman and J. J. Jasieniak, Chem. Commun., 2017, 53, 232-235.

212 Y. Tan, Y. Zou, L. Wu, Q. Huang, D. Yang, M. Chen, M. Ban, C. Wu, T. Wu, S. Bai, T. Song, Q. Zhang and B. Sun, ACS Appl. Mater. Interfaces, 2018, 10, 3784-3792.

213 D. Yang, X. Li, W. Zhou, S. Zhang, C. Meng, Y. Wu, Y. Wang and H. Zeng, Adv. Mater., 2019, 31, 1900767.

214 G. Li, J. Huang, H. Zhu, Y. Li, J.-X. Tang and Y. Jiang, Chem. Mater., 2018, 30, 6099-6107.

215 J. Xue, J.-W. Lee, Z. Dai, R. Wang, S. Nuryyeva, M. E. Liao, S.-Y. Chang, L. Meng, D. Meng, P. Sun, O. Lin, M. S. Goorsky and Y. Yang, Joule, 2018, 2, 1866-1878.

216 J. Li, L. Xu, T. Wang, J. Song, J. Chen, J. Xue, Y. Dong, B. Cai, Q. Shan, B. Han and H. Zeng, Adv. Mater., 2017, 29, 1603885.

217 Z. Yang, M. Wang, H. Qiu, X. Yao, X. Lao, S. Xu, Z. Lin, L. Sun and J. Shao, Adv. Funct. Mater., 2018, 28, 1705908.

218 R. M. Maceiczyk, K. Dümbgen, I. Lignos, L. Protesescu, M. V. Kovalenko and A. J. deMello, Chem. Mater., 2017, 29, 8433-8439.

219 M. Hao, Y. Bai, S. Zeiske, L. Ren, J. Liu, Y. Yuan, N. Zarrabi, N. Cheng, M. Ghasemi, P. Chen, M. Lyu, D. He, J.-H. Yun, Y. Du, Y. Wang, S. Ding, A. Armin, P. Meredith, G. Liu, H.-M. Cheng and L. Wang, Nat. Energy, 2020, 5, 79-88. 